NBER WORKING PAPER SERIES

\title{
RENT RIGIDITY, ASYMMETRIC INFORMATION, AND VOLATILITY BOUNDS IN LABOR MARKETS
}

\author{
Bjoern Bruegemann \\ Giuseppe Moscarini \\ Working Paper 13030 \\ http://www.nber.org/papers/w13030
NATIONAL BUREAU OF ECONOMIC RESEARCH
1050 Massachusetts Avenue
Cambridge, MA 02138 \\ April 2007
}

We thank seminar participants at the 2005 NBER Summer Institute, University of Connecticut, Yale, Hunter College, Tinbergen Institute, MIT, LABORatorio R.R., Harvard, Penn, 2006 SED, NYU and Georgetown for comments. We gratefully acknowledge insightful conversations with Dino Gerardi. The views expressed herein are those of the author(s) and do not necessarily reflect the views of the National Bureau of Economic Research.

(C) 2007 by Bjoern Bruegemann and Giuseppe Moscarini. All rights reserved. Short sections of text, not to exceed two paragraphs, may be quoted without explicit permission provided that full credit, including $\odot$ notice, is given to the source. 
Rent Rigidity, Asymmetric Information, and Volatility Bounds in Labor Markets Bjoern Bruegemann and Giuseppe Moscarini

NBER Working Paper No. 13030

April 2007

JEL No. E24,E32

\begin{abstract}
$\underline{\text { ABSTRACT }}$
Recent findings have revived interest in the link between real wage rigidity and employment fluctuations, in the context of frictional labor markets. The standard search and matching model fails to generate substantial labor market fluctuations if wages are set by Nash bargaining, while it can generate fluctuations in excess of what is observed if wages are completely rigid. This suggests that less severe rigidity may suffice. We study a weaker notion of real rigidity, which arises only in frictional labor markets, where the wage is the sum of the worker's opportunity cost (the value of unemployment) and a rent. With wage rigidity this sum is acyclical; we consider rent rigidity, where only the rent is acyclical. We offer two contributions. First, we derive upper bounds on labor market volatility that apply if the model of wage determination generates weakly procyclical worker rents, and that are attained by rent rigidity. Quantitatively, the bounds are tight: rent rigidity generates no more than a third of observed volatility, an outcome that is closer to Nash bargaining than to wage rigidity. Second, we show that the bounds apply to a sequence of famous solutions to the bargaining problem under asymmetric information: at best they generate rigid rents but not rigid wages.
\end{abstract}

Bjoern Bruegemann

Yale University

Department of Economics

PO Box 208268

New Haven, CT 06520-8268

bjoern.bruegemann@yale.edu

Giuseppe Moscarini

Yale University

Department of Economics

PO Box 208268

New Haven, CT 06520-8268

and NBER

giuseppe.moscarini@yale.edu 


\section{Introduction}

The role of real wage rigidity in shaping employment fluctuations is a perennial subject of macroeconomics. Interest in this subject has recently been revived by the finding that a conventional calibration of the baseline textbook search and matching model driven by labor productivity shocks of plausible magnitude and persistence grossly fails to account for the observed volatility of unemployment and vacancies (Shimer, 2005; Hall, 2005; Costain and Reiter, 2005). If the standard assumption of Nash-bargained wages is replaced by a rigid wage, however, then the model is capable of generating fluctuations in excess of what is observed (Hall, 2005). Thus in principle other models of wage determination may be able to match observed volatility by generating wages which are not completely yet sufficiently rigid. Specifically, Shimer (2005) asks whether bargaining under asymmetric information may enhance amplification. What characteristics must a model of wage determination have in order to match observed volatility?

In this paper we study a notion of rigidity weaker than wage rigidity. It arises naturally in frictional labor markets, where the compensation of the worker is the sum of his opportunity cost (the value of unemployed job search), and a quasi-rent that accrues when he finds a job. With wage rigidity as in Hall (2005) overall compensation is acyclical. Because the value of unemployment is higher in booms, when jobs are much easier to find, the worker's quasi-rent must be countercyclical to keep wages constant. We consider rent rigidity, where only the rent component is acyclical, and ask the following question: must a model of wage determination generate countercyclical rents to match observed volatility, or is rent rigidity sufficient?

Our answer is clear-cut: rent rigidity alone does not come close to matching observed volatility. Second, we show that many famous and quite different solutions to the wagesetting problem feature at best rent rigidity, but never wage rigidity. We put special but not exclusive emphasis on environments with asymmetric information, thereby shedding light on the question raised by Shimer. These are our two main contributions. We now provide some details.

To show that rent rigidity alone can produce only very limited amplification, we derive two upper bounds on labor market volatility, which apply if the model of wage determination generates weakly procyclical worker rents, and are attained if rents are rigid. The first, weak 
bound applies under mild regularity conditions on the model of wage determination, while the second, strong bound applies under slightly more demanding conditions. The latter has the advantage that only information from Shimer's (2005) calibration is needed to conclude that it is quantitatively tight : it allows for at most a third of observed volatility. The same conclusion obtains for the weak bound if one additional element is added to Shimer's calibration: empirical information on the average cost of hiring a worker. Thus, unlike wage rigidity, rent rigidity alone does not enable the model to generate labor market fluctuations as large as in the data.

The mechanics behind the volatility bounds are as follows. Firms must be compensated for the initial expense of hiring a worker by receiving quasi-rents over the life of the match. Assume that worker rents are acyclical, the best case scenario for amplification if they cannot be countercyclical. There are two possibilities, depending on the size of hiring costs.

First, suppose the average cost of filling a vacancy is small relative to overall gains from trade in the labor market. In this case only small rents go to firms in compensation for hiring costs, and worker rents must be large. In other words, finding a job yields unemployed workers a large capital gain, both in booms and recessions as worker rents are acyclical. Now consider an increase in the job finding rate. Since worker rents are large and constant, this has a large positive effect on the opportunity cost of the worker: although the capital gain from finding a job does not increase, it is large and more likely to accrue to the worker. Added to the constant rents from employment, this larger value of unemployment translates one to one into a strong increase in wages. Thus if an increase in productivity raises the job finding rate, then this feedback effect through wages counteracts the increase in profits and thereby mutes vacancy creation. Notice that this mechanism is similar to the reason why Nash bargaining generates little amplification, with one important difference: with Nash bargaining the feedback effect is driven both by an increase in worker rents and by an increase in the job finding rate. Rigid rents preserve only the latter effect, but this is strong enough to severely curtail amplification.

The weak bound is solely based on this mechanism, which has bite only if hiring cost are small. Evidence on hiring costs suggest that they are indeed small relative to overall gains from trade, allowing us to conclude that the weak bound is quantitatively tight.

The strong bound requires no calibration of hiring costs, because it takes into account a second effect which curtails amplification if hiring costs are large relative to overall gains from 
trade in the labor market. In this case firms must receive large quasi-rents as compensation for the initial hiring expense. Hence worker rents are small. While this weakens the feedback effect, it introduces a second effect. The matching technology is such that a vacancy is less likely to result in a match if the ratio of vacancies to unemployment in the economy is large, a congestion effect. As a consequence, an increase in the vacancy-unemployment ratio induces a proportional increase in the cost of filling a vacancy. If hiring costs are large on average, this increase is large in absolute value. More importantly, if the increase in the vacancyunemployment ratio is caused by an increase in productivity, then the increase in hiring costs is large relative to the original increase in productivity, once again muting vacancy creation.

To summarize our first contribution, rent rigidity is quantitatively much closer to Nash bargaining than it is to wage rigidity. Thus merely switching to a different model of wage determination does not allow the search and matching model to match observed volatility, unless the model of wage determination is capable of generating countercyclical worker rents.

Our second contribution is to show that rent rigidity, thus our volatility bounds, apply to an interesting class of models of wage determination. We focus on environments with asymmetric information, thereby shedding light on Shimer's question whether this can amplify the response of the model to productivity shocks. We assume that, upon being matched, the firm privately draws a match-specific productivity component and the worker a match-specific amenity value of the job. The distributions are fixed and independent of (publicly observed) aggregate labor productivity, and satisfy standard regularity conditions, but are otherwise unrestricted. In this environment, we study in detail three classical wage determination models. In the monopoly (or monopsony) solution, where either the firm or the worker makes a take-it-or-leave-it wage proposal to the other privately informed party, it follows naturally that worker rents cannot be countercyclical. If the firm is making the offer, it becomes more eager to trade in a boom, and as a consequence it will concede more informational rents to the worker. If the worker is making the offer, then in a boom once again firms are more eager to trade, thus the worker is in a better position to extract rents through more aggressive wage requests. In both cases the most that can be hoped for is no increase in worker rents in a boom. This basic intuition carries over when we relax the commitment assumptions associated with take-it-or-leave-it offers, and study strategic bargaining, both repeated one-sided offers (Coase conjecture) and alternating offers. Finally, in an environment with bilateral asymmetric information, we consider the 
constrained efficient allocation, obtained with the help of a mediator (e.g. an arbitrator in wage contracting), as in Myerson and Satterthwaite (1983). Again we show that rent rigidity is the most that can be obtained, for a similar reason: in a boom the mediator desires to induce more trade, and in order to do so he has to increase informational rents going to workers.

To summarize our second contribution, at the very least asymmetric information introduces, through the distribution of types, many additional degrees of freedom that can be exploited to generate wage rigidity. In the models we study, however, these degrees of freedom are not enough to match observed labor market volatility.

Others have taken up Shimer's question and investigated whether asymmetric information enables the search and matching model to produce larger labor market fluctuations. Guerrieri (2006) studies competitive search equilibrium, whereas we analyze bargaining games with random matching, making the two analyses complementary. The approaches are different, however. She simulates the model for some specific distributions of types. We derive labor market volatility bounds that apply for any distribution of types. In fact, one can show that our bounds also apply to her model. Her results are consistent with ours: she finds very little amplification. Kennan (2006) and Menzio (2005b) take a different route, relying on exogenous fluctuations in the shape of the distribution of types as the key driving force of labor market fluctuations. In contrast, Guerrieri's and this paper keep the extent of asymmetric information itself acyclical, examining how the presence of asymmetric information amplifies the response of the model to a productivity shock.

In Section 2 we introduce the economy. The labor market volatility bounds are derived in Section 3. In Section 4 we verify these bounds for the models of wage bargaining under asymmetric information. For simplicity, the analysis in Sections 2-4 restricts attention to permanent labor productivity shocks. A fortiori, our results also apply to transitory shocks, which generate a weaker response of the labor market. For completeness, Section 5 formally derives the bounds for the case of transitory shocks, and provides an illustration using the monopoly model. Section 6 concludes. 


\section{The Economy}

We consider the baseline search-and-matching model employed by Shimer (2005). We extend it to allow for bilateral asymmetric information about match-specific values: the worker may have private information about how much he likes the job, and the firm may have private information about productivity.

\subsection{Preferences and Technology}

The economy is populated by a measure 1 of workers and a much larger measure of firms. All agents are infinitely-lived, risk neutral and share the discount rate $r>0$. Workers can either be employed or unemployed. An unemployed worker receives flow utility $b$ and searches for a job. Employed workers receive endogenously determined wage payments from their employers and cannot search for other jobs. Firms can search for a worker by maintaining an open vacancy at flow cost $c$. Free entry implies that the value of an open vacancy is zero. Unemployed workers $u$ and vacancies $v$ are matched at rate $m(u, v)$ where $m$ is a constant returns to scale matching function. Let $\theta \equiv \frac{v}{u}$ denote the vacancy-unemployment ratio. Then vacancies are matched at rate $m(1 / \theta, 1) \equiv q(\theta)$ and workers are matched at rate $m(1, \theta)=\theta q(\theta)$.

Upon being matched, the worker draws a match specific amenity value $z$ from the distribution $F_{Z}$ and the firm draws a match specific productivity component $y$ from the distribution $F_{Y}$. The draws are once and for all until the match dissolves. Without loss in generality, the two distributions have mean zero. Output of the match is given by $p+y$, so $p$ is ex ante average labor productivity. However, in general, not all matches are formed and $p$ will not equal labor productivity averaged across existing matches. We will refer to $p$ as the aggregate component of labor productivity. The amenity value $z$ adds to the wage to determine the flow value of employment for the worker. This value $z$ may be private information of the worker, and the idiosyncratic productivity component $y$ may be private information of the firm. Matches are destroyed exogenously at rate $\delta$.

We want to study the magnitude of the response of the labor market to aggregate labor productivity shocks. One way to proceed would be to specify a stochastic process for labor productivity and evaluate the labor market volatility it induces. But to obtain upper bounds on labor market volatility we can simply examine the response of the model to a permanent 
unanticipated productivity shock. This response provides an upper bound to the volatility induced by a stochastic process with transitory shocks for an intuitive reason: a given increase in productivity increases incentives to create jobs more the longer it is expected to last. ${ }^{1}$ Of course, this simpler approach introduces some slack and it may be possible to obtain tighter bounds which explicitly account for the degree of persistence of productivity shocks. However, due to the strong persistence of labor productivity in US data, the gain from such a tighter bound would be quantitatively small. Thus the analysis in Sections 2-4 analyzes the case of permanent productivity shocks, as the the cost of doing so is small relative to the gains in terms of both simplicity and transparency of the analysis. In Section 5 we generalize the analysis to the case of transitory shocks.

\subsection{Wage Determination}

Due to search frictions, a match generates quasi-rents. Thus we need to make assumptions regarding how these rents are split between the worker and the firm. In other words, we need a model of wage determination: a mapping of the relevant variables that describe the environment of the match into a decision whether to trade and how to split the quasi-rents associated with the match. We work with a general definition of a model of wage determination, because our objective is to obtain volatility bounds that apply to entire classes of models of wage determination.

Here the assumption that productivity shocks are unanticipated and permanent keeps matters simple: the environment of the match is characterized by only two aggregate variables. The first is productivity $p$, which is expected to be constant over the life of the match. The second variable is the endogenous opportunity cost of the worker, which is given by the utility associated with unemployment $U$. It will be convenient to let $n=r U$ represent the opportunity cost of the worker in flow terms. This opportunity cost is a jump variable

\footnotetext{
${ }^{1}$ An extreme example may help in building intuition. Suppose matches last for several years on average. Moreover, suppose that the economy switches between boom and recession every week. Clearly matches created in booms and recessions are essentially the same, and the additional incentives to create matches in booms are minimal relative to the case in which the economy switches from boom to recession only every few years. See Section 5 as well as Mortensen and Nagypal (2005) and Hornstein, Krusell, and Violante (2005) for a more formal development of this point.
} 


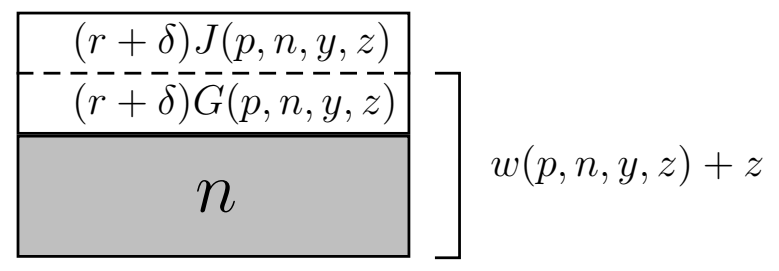

and constant along the equilibrium path of the economy following a productivity shock, a convenient feature of the standard search and matching model.

For each pair of match-specific values $y$ and $z$, a model of wage determination maps the two variables $p$ and $n$ into a trade decision and a split of quasi-rents. It is important to emphasize that the timing of wage payments over the life of a match is of no importance for labor market fluctuations. Only the present value of rents going to the worker and the firm matter. Thus we formulate a model of wage determination directly in terms of the present values of rents.

Let $G(y, z, p, n)$ denote the present value of rents going to the worker, $J(y, z, p, n)$ denote the present value of rents going to the firm, and $x(y, z, p, n)$ be the probability that a match is formed conditional on the realizations $y$ and $z$.

Definition 1. A model of wage determination is a triple of functions $\{G, J, x\}$ mapping $\mathbb{R}^{4}$ into $\mathbb{R}_{+}^{2} \times[0,1]$ and satisfying

$$
(r+\delta)[G(y, z, p, n)+J(y, z, p, n)]=x(y, z, p, n)(y+z+p-n) .
$$

In terms of standard notation, the overall payoff of the employed worker is $W=G+U$, so $G=W-U$ is the familiar capital gain an unemployed worker receives from being hired. Although the timing of wage payments over the life of the match is not important, in order to provide a link with wages, one can define the annuity value of the present value of wages $w(p, n, y, z)=(r+\delta) G(y, z, p, n)+n-z$, which would be the level of the wage if payments were constant over the life of the match. Figure 1 illustrates the concept of a model of wage determination. The entire bar represents the value of the match in flow terms, namely 
$p+y+z-b$. The gray bar represents the opportunity cost of the worker $n$, and the white bar the quasi-rents which are split between the firm and the worker. The annuity value of profits is $(r+\delta) J(y, z, p, n)$, the annuity value of rents going to the worker is $(r+\delta) G(y, z, p, n)$. Adding the opportunity cost $n$ to the latter and subtracting the amenity value of the job $z$ yields the annuity value of wages $w(p, n, y, z)$.

We illustrate this concept with two specific models which will serve as important benchmarks throughout the paper.

Nash bargaining. Under Nash bargaining as commonly employed in the textbook search and matching model, quasi-rents of the match are shared between the worker and the firm with shares $\beta$ and $1-\beta$, respectively: in flow terms

$$
\begin{aligned}
& (r+\delta) G(y, z, p, n)=x(y, z, p, n) \beta(p-n+y+z) \\
& (r+\delta) J(y, z, p, n)=x(y, z, p, n)(1-\beta)(p-n+y+z) .
\end{aligned}
$$

The probability of trade is one if forming the match is ex post efficient, zero otherwise: $x(y, z, p, n)=\mathbb{I}\{p-n+y+z \geq 0\}$ where $\mathbb{I}$ is an indicator function.

Wage Rigidity (Hall (2005)). Hall considers a model without heterogeneity in $y$ and $z$. Trade is bilaterally efficient, that is $x(p, n)=\mathbb{I}\{p-n \geq 0\}$. Wages are rigid in the following sense: the annuity value of wages received by the worker in a new match $\bar{w}$ does not respond to changes in $p$ and $n$, as long as this does not interfere with efficient trade:

$$
(r+\delta) G(p, n)=\left\{\begin{array}{cl}
\bar{w}-n & p \geq \bar{w} \geq n, \\
p-n & \bar{w}>p \geq n, \\
0 & \text { otherwise }
\end{array} \quad(r+\delta) J(p, n)=\left\{\begin{array}{cc}
p-\bar{w} \quad p \geq \bar{w} \geq n \\
p-n \quad p \geq n>\bar{w} \\
0 & \text { otherwise }
\end{array}\right.\right.
$$

As discussed before, the timing of wage payments through which the annuity value $\bar{w}$ is implemented is not essential here.

We now use the graphical apparatus introduced in Figure 1 to compare these two models of wage determination. In doing so, we also introduce the key distinction between the two notions of rigidity contrasted in this paper: wage rigidity vs. rent rigidity. The basic idea is illustrated in Figure 2, where we abstract from match-specific draws $y$ and $z$ for simplicity. 
Figure 2: Wage Rigidity vs. Rent Rigidity

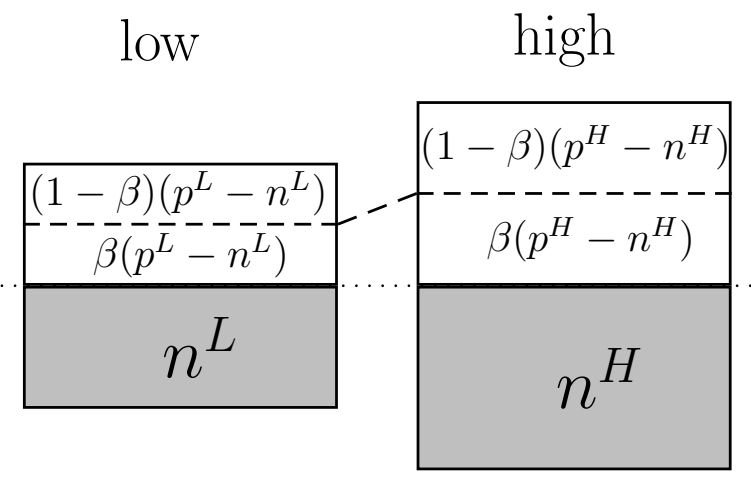

(a) Nash Bargaining

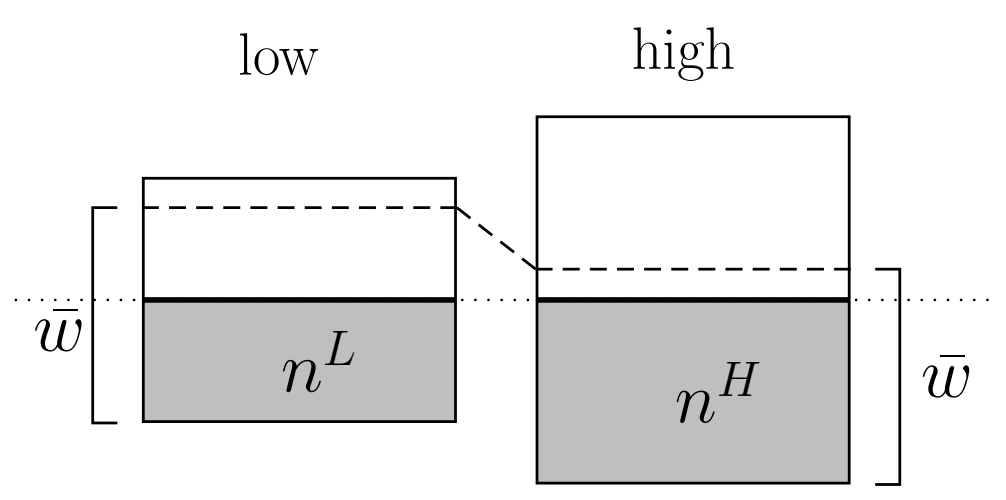

(b) Wage Rigidity

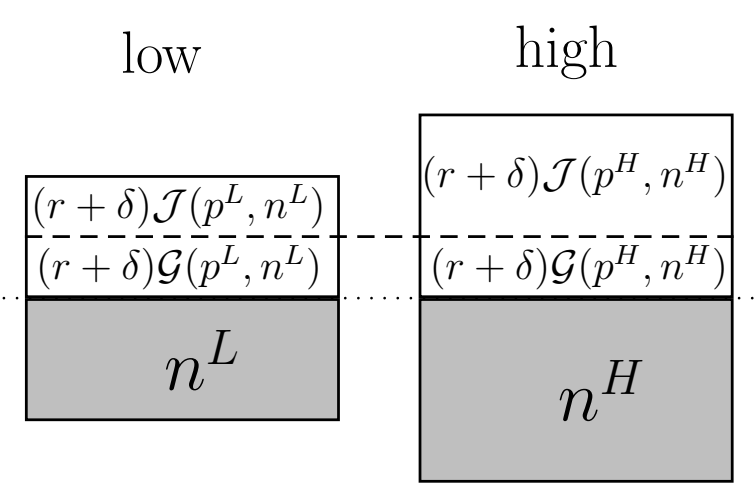

(c) Rent Rigidity 
Each panel encompasses two replicas of Figure 1, one for a low aggregate state $p^{L}$ and one for a high aggregate state $p^{H}>p^{L}$.

Panel (a) displays the case of Nash Bargaining. The quasi-rent is split with fixed shares $\beta$ and $(1-\beta)$. As the overall quasi-rent is procyclical, it follows that the rent going to the worker is also procyclical. ${ }^{2}$

Panel (b) shows the case of wage rigidity. Even with rigid wages the opportunity cost of workers are procyclical in the stochastic equilibrium of the economy. ${ }^{3}$ As shown in the figure, this implies that rents must be countercyclical.

Panel (c) illustrates the contrast with the notion of rent rigidity. Here the rent going to the worker does not increase in a boom, but the annuity value of wages $w(p, n)$ increases because the opportunity cost of the worker $n$ does. In this sense, rent rigidity is weaker than wage rigidity.

It is instructive to relate rent rigidity to Nash bargaining with an ad hoc countercyclical bargaining share $\beta(p)$. Shimer (2005) shows in a reduced form experiment that making the bargaining share somewhat countercyclical helps in generating more volatile unemployment and vacancies. We emphasize that a countercyclical bargaining share per se does not imply countercyclical rents. It is clear from Figure 2 (c) that if the bargaining share is only slightly countercyclical, then rents remain procyclical. As one continues to make the bargaining share more countercyclical, eventually the qualitative threshold will be crossed at which rents also become countercyclical. One way of putting the question we will answer in the next section is then as follows: do we need to cross this threshold in order to generate the observed extent of volatility?

\subsection{Equilibrium Conditions}

Having defined models of wage determination we can now write down the equilibrium conditions of the model. Before doing so, it is useful to make precise how we will evaluate the extent of labor market volatility generated by the model. We follow the literature and focus on the

\footnotetext{
${ }^{2}$ Procyclicality of the overall quasi-rent is an equilibrium implication of the standard search and matching model with Nash bargaining, which we discuss in detail in Section 3.

${ }^{3}$ As we will show in Section 3, in a boom, even though unemployed workers do not get a higher wage should they find a job, they are more likely to find a job, so the value of unemployment increases.
} 
vacancy-unemployment ratio $\theta=\frac{v}{u}$ rather than unemployment and vacancies separately. In US data, detrended vacancies and unemployment are strongly negatively correlated, so their ratio is especially volatile. Thus the volatility of the $v$-u ratio presents a convenient way of capturing the challenge facing the model in a single statistic. ${ }^{4}$

The model has two endogenous variables: the $v-u$ ratio $\theta$ and the opportunity cost of the worker $n$. Both immediately jump to their new steady state values in response to a permanent productivity shock. ${ }^{5}$ Thus one can compute the elasticity $\varepsilon_{\theta}$ of the $v$ - $u$ ratio with respect to a productivity shock by differentiating the steady state equations of the model.

Given a model of wage determination, we can compute the ex ante (before matching) averages of rents and the probability of trade

$$
\begin{aligned}
\mathcal{G}(p, n) & \equiv \iint G(y, z, p, n) d F_{Y}(y) d F_{Z}(z), \\
\mathcal{J}(p, n) & \equiv \iint J(y, z, p, n) d F_{Y}(y) d F_{Z}(z), \\
\xi(p, n) & \equiv \iint x(y, z, p, n) d F_{Y}(y) d F_{Z}(z) .
\end{aligned}
$$

With this notation we study two steady state conditions in $(\theta, n) .{ }^{6}$ First, the free entry condition, equating the flow cost of posting a vacancy $c$ to the expected capital gain, which is the rate $q(\theta)$ at which open vacancies meet a worker, times the expected value $\mathcal{J}(p, n)$ to the firm of meeting a worker, taking into account that the match may potentially fail to form:

$$
c=q(\theta) \mathcal{J}(p, n)
$$

Second, the equation determining the flow value of unemployment as the flow value of leisure $b$ plus the expected capital gain, the rate $\theta q(\theta)$ at which unemployed workers contact open vacancies times the expected return $\mathcal{G}(p, n)$ from the contact, again taking into account that the match may potentially fail to form:

$$
n=b+\theta q(\theta) \mathcal{G}(p, n) .
$$

\footnotetext{
${ }^{4}$ Moreover, as discussed by Shimer (2005) the model already does reasonably well at capturing comovement and relative volatility of unemployment and vacancies. In this sense there is little need to examine unemployment and vacancies separately, and it is sufficient to focus on the volatility of the $v$ - $u$ ratio.

${ }^{5}$ Pissarides (2000), pp. 26-33.

${ }^{6}$ Once $\theta=\frac{v}{u}$ has been computed from these conditions, steady state levels of unemployment $u$ and then vacancies $v=\theta u$ are computed using the Beveridge curve $u=\frac{\delta}{\delta+\theta q(\theta)}$. Since we focus on the $v$ - $u$ ratio we do not make use of this relationship.
} 


\section{Labor Market Volatility Bounds}

In this section we derive two bounds on labor market volatility and evaluate them quantitatively. These bounds apply to models of wage determination which generate weakly procyclical, that is at best rigid but not countercyclical, worker rents.

So far we have not put any structure on the three functions that make up the model of wage determination. We now introduce a set of regularity conditions. They are quite weak and are satisfied by all models of wage determination considered in this paper, including Nash bargaining and wage rigidity. This is important since we are interested in models of wage determination which lie in some sense in between these two benchmark models.

Condition 1. The model of wage determination $\{G, J, x\}$ satisfies three properties:

(a) The partial effect of aggregate labor productivity $p$ on ex ante worker rents is nonnegative: for all $p, n$,

$$
\frac{\partial \mathcal{G}(p, n)}{\partial p} \geq 0
$$

(b) The partial effect of the worker's opportunity cost $n$ on ex ante firm rents is non-positive: for all $p, n$,

$$
\frac{\partial \mathcal{J}(p, n)}{\partial n} \leq 0
$$

(c) The trade probability $x(p, n, y, z)$ depends on $p$ and $n$ only through their difference $p-$ $n$, and is weakly increasing in both match-specific outcomes, the firm's idiosyncratic productivity component $y$ and the worker's amenity value for the job $z$.

Part (a) requires that holding worker opportunity cost $n$ constant, an increase in labor productivity will not reduce worker rents. Importantly, this does not imply procyclical worker rents: under wage rigidity the partial effect of labor productivity is exactly zero; thus worker rents are countercyclical as worker opportunity $\operatorname{costs} n$ increase in booms. Similarly, part (b) does not imply that firm profits are countercyclical. Again wage rigidity represents the extreme case in which the partial effect is zero; firm rents under wage rigidity are then procyclical as the productivity component $p$ is higher in booms.

Both Nash bargaining and wage rigidity feature ex post efficient trade, that is trade occurs if and only if $p-n+y+z \geq 0$. Ex post efficiency immediately implies part (c). The converse 
is not true, that is part (c) is weaker than ex post efficiency. We will see that part (c) holds for all the models featuring ex post inefficient trade considered in Section 4. For an interpretation of this property, first consider comparative statics with respect to $p$ and $n$. If $p$ and $n$ increase by the same amount, this shifts up the location of the bargaining problem to a higher level of productivity and opportunity cost, but leaves the average gains from trade $p-n$ unchanged. Condition 1 (c) requires that such a shift leaves the probability of trade unaffected. The second requirement of Condition 1 (c) specifies that matches are positively selected. Let $\bar{y}(p, n) \equiv \xi(p, n)^{-1} \iint x(p, n, y, z) y d F_{Y}(y) d F_{Z}(z)$ be the average match specific productivity component conditional on trade. Condition 1 (c) implies $\bar{y}(p, n) \geq 0 .{ }^{7}$ Similarly, it implies that the conditional mean amenity value $\bar{z}(p, n)$ is non-negative.

We now derive two bounds on labor market volatility, referred to as weak and strong, respectively. The weak bound is less tight theoretically, and more empirical information must be utilized in order to conclude that it is tight quantitatively. The second bound holds under more stringent conditions on the model of wage determination.

\subsection{Weak Labor Market Volatility Bound}

The first bound applies to all models of wage determination that satisfy the following property (along with regularity Condition 1).

Definition 2. (Increasing Worker Rents) In a model of wage determination $\{G, J, x\}$, worker rents are Increasing if, for all $p, n$,

$$
\frac{\partial \mathcal{G}(p, n)}{\partial p} \geq-\frac{\partial \mathcal{G}(p, n)}{\partial n} \geq 0
$$

Notice that this property is stronger than Condition 1 (a): the partial effect of aggregate labor productivity $p$ on worker rents is not only non-negative, but it is required to exceed in absolute value the effect of the worker's opportunity cost $n$ on worker rents.

To explain the role of Increasing worker rents, it is useful to define the following property of the equilibrium.

Definition 3. (Procyclical Worker Rents) Worker rents are Procyclical in equilibrium

\footnotetext{
${ }^{7}$ Recall that the unconditional mean of match specific productivity has been normalized to zero.
} 
if

$$
\frac{d \mathcal{G}(p, n)}{d p}=\frac{\partial \mathcal{G}(p, n)}{\partial p}+\frac{\partial \mathcal{G}(p, n)}{\partial n} \frac{d n}{d p} \geq 0
$$

As will emerge clearly from the derivation, the weak labor market volatility bound applies whenever worker rents are Procyclical in equilibrium. Procyclicality, however, is a feature of the equilibrium and not a condition that can be verified only examining the model of wage determination. Increasing worker rents is a sufficient condition on the model of wage determination which insures that worker rents are Procyclical in equilibrium.

We obtain the volatility bound for $\theta$ in three steps. First we show that if worker rents are Procyclical and the elasticity $\varepsilon_{\theta}=d \ln \theta / d \ln p$ has to be positive, then the opportunity cost of the worker responds less than one for one to an increase in productivity, that is $\frac{d n}{d p}<1$. Second we transform this inequality for $\frac{d n}{d p}$ into the desired upper bound on $\varepsilon_{\theta}$, which applies whenever worker rents are Procyclical. Finally we show that if worker rents are Increasing, then they are Procyclical.

First, we show that $\varepsilon_{\theta}>0$ implies $\frac{d n}{d p}<1$. If the increase in the outside option of the worker in a boom was so large as to overturn the increase in productivity, then firm rents would shrink, which is inconsistent with more intensive hiring activity of firms. We refer to $\frac{\partial \mathcal{J}}{\partial n} \frac{d n}{d p}$ as the feedback effect, as it captures how an increase in profits that raises vacancies and thereby the job finding rate feeds back negatively into profits through a higher opportunity cost of workers. To see that firm profits must shrink, namely $\frac{d \mathcal{J}}{d p} \leq 0$, if $\frac{d n}{d p} \geq 1$, first suppose that $\frac{\partial \mathcal{J}}{\partial p} \leq 0$. Then both the increase in productivity and - by virtue of part Condition 1 (b) - the increase in $n$ reduce firm profits. If instead $\frac{\partial \mathcal{J}}{\partial p} \geq 0$ it follows from parts (a) and (c) of Condition 1 that the sum of worker and firm rents must be increasing in $p-n$. In this case $\frac{d n}{d p} \geq 1$ implies that total rents fall, and Procyclical worker rents imply that once again firm rents must decrease. Through the entry condition (2) lower firm rents in turn imply that labor market tightness must fall, contradicting $\varepsilon_{\theta}>0$.

Second, from Equation (3), it is clear that an increase in worker rents raises the opportunity $\operatorname{cost} n$ : a larger capital gain from finding a job increases the utility of unemployed workers. Specifically, Procyclical worker rents and Equation (3) imply the following lower bound on $\frac{d n}{d p}:$

$$
\frac{d n}{d p} \geq \eta \varepsilon_{\theta} \theta q \frac{\mathcal{G}}{p}
$$


Here $\eta \equiv 1-\frac{d q}{d \theta} \frac{\theta}{q}$ denotes the elasticity of the matching function with respect to vacancies, evaluated at the initial equilibrium. Similarly, $\theta, q$, and $\mathcal{G}$ are the $v$ - $u$ ratio, the contact rate of vacancies, and worker rents, all evaluated at the initial equilibrium. Inequality (4) holds as an equality if worker rents are completely rigid. Notice that even in that case $\frac{d n}{d p}$ is positive: the value of unemployment increases because it is easier to find a job. Moreover, a large elasticity $\varepsilon_{\theta}$ implies that the job finding rate responds strongly to an increase in productivity, leading to a larger value of $\frac{d n}{d p}$. Combining (4) with $\frac{d n}{d p}<1$ from the first step implies an upper bound on the elasticity $\varepsilon_{\theta}$

$$
\varepsilon_{\theta} \leq \frac{1}{\eta} \frac{1}{\theta q} \frac{p}{\mathcal{G}} .
$$

The interpretation of this upper bound is straightforward. If $\eta$ is large, then the job finding rate responds strongly to an increase in $\theta$ because vacancies are very productive in the creation of matches. This is associated with a stronger feedback effect and leads to a tighter bound on $\varepsilon_{\theta}$. If the job-finding rate $\theta q$ is larger, then a given percentage increase in $\theta$ translates into a larger increase in the chances of finding a job, also tightening the bound. Finally, the bound is tighter if the level of rents going to the worker is higher. If the capital gain of finding a job is zero, then a higher job-finding rate does not translate into a higher value of unemployment because there is nothing to be gained from finding a job.

To turn (5) into a bound that can be evaluated quantitatively we need to calibrate worker rents $\mathcal{G}$. For this purpose we introduce some additional notation. First the annuity value of wages in the initial equilibrium is $w \equiv(r+\delta) \xi^{-1} \mathcal{G}+n-\bar{z}$ (recall that the average amenity value $\bar{z}$ must be deducted from the average flow utility of an employed worker in order to obtain wages). Next define the job finding rate

$$
f \equiv \theta q \xi
$$

which is the product of the matching rate $\theta q$ and the probability of match formation $\xi$. Finally define observed average labor productivity, the average of $p+y$ conditional on trade $\bar{p} \equiv p+\bar{y}$. Using this notation together with Equation (3) we can rewrite (5) as

$$
\varepsilon_{\theta} \leq \frac{\bar{p}-\bar{y}}{w+\bar{z}-b} \frac{1}{\eta} \frac{r+\delta+f}{f} .
$$

At this point we use Condition 1 (c): the fact that matches that do form are positively selected 
implies that $\bar{y} \geq 0$ and $\bar{z} \geq 0$. This yields

$$
\varepsilon_{\theta} \leq \frac{\bar{p}}{\bar{p}-b} \frac{\bar{p}-b}{w-b} \frac{1}{\eta} \frac{r+\delta+f}{f} \equiv \bar{\varepsilon}_{\theta, W E A K}
$$

The third and final step is to show that if worker rents are Increasing, then they are Procyclical. Suppose not. This can only happen if $\frac{d n}{d p}>1$. Increasing worker rents in conjunction with Condition 1 (c) imply $\frac{\partial \mathcal{J}}{\partial p}+\frac{\partial \mathcal{J}}{\partial n} \leq 0$. Thus $\frac{d n}{d p}>1$ and Condition 1 (b) imply that firm rents decrease in response to an increase in $p$. For firms to be willing to post vacancies in the face of lower rents the $v$ - $u$ ratio must fall, that is equilibrium condition (2) implies $\varepsilon_{\theta} \leq 0$. But with both worker rents and the job finding rate countercyclical it follows from Equation (3) that the utility of unemployed workers must be lower, that is $\frac{d n}{d p} \leq 0$, a contradiction.

We have established the following:

Proposition 1. (Weak Volatility Bound) If the model of wage determination satisfies Condition 1 and worker rents are Procyclical, then $\varepsilon_{\theta} \leq \bar{\varepsilon}_{\theta, W E A K}$. If worker rents are Increasing, then they are Procyclical.

Our approach in Section 4 is to show that a given model of wage determination under asymmetric information satisfies both Condition 1 and Increasing worker rents. Proposition 1 then allows us to conclude that this model of wage determination is subject to the weak labor market volatility bound. Proposition 1 can also be applied in a different way: if a model of wage determination satisfies Condition 1 but generates amplification in excess of the bound, then it must produce countercyclical worker rents. That is, finding a job yields a larger gain in a slump. We illustrate this application with the following model of wage determination.

Outside Option Principle. Hall and Milgrom (2007) consider the bargaining theory of Binmore, Rubinstein, and Wolinsky (1986). According to this theory, the relevant threat point of the worker is not unemployment but delay of bargaining, say a strike. Let $b^{s}$ be the flow payoff of the worker during a strike, which could be different from the flow payoff during unemployment $b$. The game has a unique subgame perfect equilibrium which yields a wage $w(p, n)=b^{s}+\frac{1}{2}\left(p-b^{s}\right)$. This is similar in form to the Nash bargaining wage, but with the strike value $b^{s}$ replacing the opportunity $\operatorname{cost} n$. The model is given by $x(p, n)=\mathbb{I}\{p-n \geq 0\}$ 
and

$$
\begin{gathered}
(r+\delta) G(p, n)=\left\{\begin{array}{cc}
\frac{1}{2}\left(p+b^{s}\right)-n & \frac{1}{2}\left(p+b^{s}\right) \geq n, \\
0 & \text { otherwise }
\end{array}\right. \\
(r+\delta) J(p, n)=\left\{\begin{array}{cc}
\frac{1}{2}\left(p-b^{s}\right) & \frac{1}{2}\left(p+b^{s}\right) \geq n, \\
p-n & p \geq n \geq \frac{1}{2}\left(p+b^{s}\right), \\
0 & \text { otherwise }
\end{array}\right.
\end{gathered}
$$

Condition 1 is easily verified, but since $\frac{\partial \mathcal{G}}{\partial p}+\frac{\partial \mathcal{G}}{\partial n}=-\frac{1}{2}$ worker rents are not Increasing. Moreover, with an elasticity

$$
\varepsilon_{\theta, H M}=\frac{1}{1-\eta} \frac{p}{p-b^{s}}
$$

the model can generate a volatility exceeding the bound $\bar{\varepsilon}_{\theta, W E A K}$, by choosing $b^{s}$ sufficiently close to $p$. However, Proposition 1 allows us to conclude that the only way this can occur is if the model generates countercyclical worker rents in equilibrium. This is not obvious, as in principle the model can generate procyclical worker rents if $b^{s}$ is sufficiently close to $b .^{8}$

\subsection{Strong Labor Market Volatility Bound}

We now derive a second volatility bound which is stronger in that it uses more theory, is qualitatively tighter. Moreover, less empirical information is required to conclude that it is quantitatively tight.

As the starting point, notice that the derivation of the weak bound was based almost entirely on the second equilibrium condition (3). The entry condition (2) was used only to a limited extent, to show that a more than one for one response of opportunity costs $\frac{d n}{d p} \geq 1$ is inconsistent with a positive elasticity $\varepsilon_{\theta}$. In particular, the weak bound is based on the feedback effect arising from condition (3). As we discussed this effect is strong if the annuity value of wages and thus worker rents are large. As the annuity value of wages approaches $b$ (or equivalently worker rents approach zero) the feedback effect vanishes and the bound becomes very loose, asymptoting to infinity.

We now show that if worker rents are small, then a second effect neglected by the weak bound comes into play and limits amplification. The strong bound is obtained by fully exploiting the entry condition. The mechanics are as follows. If total rents from a match are

\footnotetext{
${ }^{8}$ One can check that worker rents are procyclical for $b=b^{s}$ under Shimer's calibration.
} 
not negligible and worker rents are small, then firm rents are large. Because of free entry, firm rents exactly equal the expected cost of hiring a worker. This cost is given by $\frac{c}{q \xi}$, that is the flow cost multiplied by the average duration of a vacancy. Notice that an increase in the $v-u$ ratio makes the recruiting effort of firms less effective due to a congestion effect: an increase in $\theta$ by one percent increases hiring costs by $1-\eta$ percent. Thus if firm rents and thereby hiring costs are large and if $\theta$ is strongly procyclical, then the increase in hiring costs in a boom will be large both in absolute terms and relative to the increase in productivity. For this to be consistent with the entry condition it must be that firm rents are highly responsive to productivity, that is $\frac{\partial \mathcal{J}}{\partial p}$ must be large. However, it is easy to see that under both wage rigidity and Nash bargaining the response of flow firm rents $(r+\delta) \frac{\partial \mathcal{J}}{\partial p}$ cannot exceed the probability of trade. This motivates the following regularity condition.

Condition 2. In a model of wage determination $\{G, J, x\}$ firm rents satisfy the property

$$
(r+\delta) \frac{\partial \mathcal{J}(p, n)}{\partial p} \leq \xi(p, n)
$$

To see that this condition holds in the case of wage rigidity, notice that the probability of trade is $\xi=1$. Since $(r+\delta) \frac{\partial \mathcal{J}}{\partial p}=1$ Condition 2 holds with equality. For Nash bargaining $(r+\delta) \frac{\partial \mathcal{J}}{\partial p}=(1-\beta) \xi$, so Condition 2 holds with some slack. More generally, notice that ex post efficient trade implies $(r+\delta)\left(\frac{\partial \mathcal{J}}{\partial p}+\frac{\partial \mathcal{G}}{\partial p}\right)=\xi$. This follows from the envelope theorem which implies that the indirect effect of an increase in $p$ through the adjustment of the trading decision is zero. Thus Condition 2 follows from ex post efficient trade in conjunction with Condition 1 (a). In this case Condition 2 is innocuous. Moreover, we will see that Condition 2 also holds for some models with asymmetric information despite ex post inefficient trade. But while Condition 1 applies to all models of wage determination considered in this paper, we do find some cases in which Condition 2 fails. Thus the first bound retains a separate role to deal with such cases.

To derive the strong bound we log-differentiate the system of Equations (2)-(3) with respect to productivity $p$ and solve for the elasticity $\varepsilon_{\theta}$ :

$$
\varepsilon_{\theta}=\frac{\bar{p}-\bar{y}}{\phi(\bar{p}-w)(1-\eta)+(w+\bar{z}-b) \eta \frac{f}{r+\delta+f}}
$$


where the term

$$
\phi \equiv \frac{\xi+f \frac{\frac{\partial \mathcal{G}}{\partial p}+\frac{\partial \mathcal{G}}{\partial n} \frac{d n}{d p}}{1-\frac{d n}{d p}}}{(r+\delta)\left(\frac{\partial \mathcal{J}}{\partial p}+\frac{\partial \mathcal{G}}{\partial p}-\frac{\frac{\partial \mathcal{G}}{\partial p}+\frac{\partial \mathcal{G}}{\partial n} \frac{d n}{d p}}{1-\frac{d n}{d p}}\right)}
$$

equals one if the equilibrium exhibits acyclical worker rents and if the trading decision is ex post efficient. Thus deviations of $\phi$ from one capture departures from these two properties. We first show that Increasing worker rents in conjunction with Condition 2 implies $\phi \geq 1$. First recall from Proposition 1 that if worker rents are Increasing, then they are Procyclical. This allows us to bound the numerator in Equation (6) from below by $(r+\delta) \frac{\partial \mathcal{J}}{\partial p}$. Moreover, this also implies $\frac{\partial \mathcal{G}}{\partial p}-\frac{\frac{\partial \mathcal{G}}{\partial p}+\frac{\partial \mathcal{G}}{\partial n} \frac{d n}{d p}}{1-\frac{d n}{d p}} \leq 0$. Together with Condition 2 this insures that the denominator is bounded from above by $\xi$. Thus $\phi \geq 1$ as desired. When this is combined with positive selection $\bar{z} \geq 0$ and $\bar{y} \geq 0$ from Condition 1 (c), then Equation (6) yields the strong volatility bound

$$
\varepsilon_{\theta} \leq \frac{\bar{p}}{\bar{p}-b}(\underbrace{\frac{\bar{p}-w}{\bar{p}-b} \frac{1}{\frac{1}{1-\eta}}}_{\text {congestion }}+\underbrace{\frac{w-b}{\bar{p}-b} \frac{1}{\frac{1}{\eta} \frac{r+\delta+f}{f}}}_{\text {feedback }})^{-1} \equiv \bar{\varepsilon}_{\theta, S T R O N G} .
$$

The first term $\frac{\bar{p}}{\bar{p}-b}$ of the product in Equation (7) captures the percentage increase in the flow gain from market activity $\bar{p}-b$ associated with a percentage increase in labor productivity $\bar{p}$. If $b$ is close to $\bar{p}$, then the flow gains from market activity are very elastic with respect to productivity. Thus everything else equal a large $b$ enables the model to generate large fluctuations in the $v-u$ ratio.

Next consider the second term, the harmonic weighted mean of the two terms $\frac{1}{1-\eta}$ and $\frac{1}{\eta} \frac{r+\delta+f}{f}$. The latter is familiar from the first bound and captures the feedback effect. The weight on this term in the mean is the fraction of the gains from market activity going to the worker $\frac{w-b}{\bar{p}-b}$ : as was reflected in the first bound, the feedback effect puts a tighter limit on amplification if the annuity value of wages is large. The first term of the weighted average $\frac{1}{1-\eta}$ is new and captures the congestion effect. This effect is more severe if $\eta$ is low, that is if an increase in the $v$ - $u$ ratio has a strong negative effect on the rate at which vacancies contact workers. The weight on this term is the fraction of the gains from market activity going to firms. Recall that this fraction is large precisely when the level of hiring costs is large. A large level of hiring costs combined with a low $\eta$ means that the absolute increase in hiring costs in a boom is large relative to the increase in productivity, putting a tight limit on amplification. 
We have established the following: ${ }^{9}$

Proposition 2. (Strong Volatility Bound) If the model of wage determination satisfies Conditions 1 and 2 and if worker rents are Increasing, then $\varepsilon_{\theta} \leq \bar{\varepsilon}_{\theta, S T R O N G}$.

We now show how the strong bound can be attained. This is of theoretical interest because one would like to know whether the bound is tight for the class of models of wage determination satisfying Conditions 1 and 2. It will also turn out to be of some interest quantitatively to the extent that the bound allows for some amplification vis-a-vis Nash bargaining.

Complete Rent Rigidity. Consider the following mode of wage determination. Trade is bilaterally efficient, that is $x(p, n)=\mathbb{I}\{p-n \geq 0\}$. The worker receives a fixed flow rent $g>0$ which does not respond to changes in $p$ and $n$, unless it would interfere with efficient trade:

$$
(r+\delta) G(p, n)=\left\{\begin{array}{cc}
g & p \geq n+g, \\
p-n & n+g>p \geq n, \\
0 & \text { otherwise }
\end{array} \quad(r+\delta) J(p, n)=\left\{\begin{array}{cc}
p-n-g & p \geq n+g \\
0 & \text { otherwise }
\end{array}\right.\right.
$$

Notice that worker rents are acyclical by construction. Conditions 1 and 2 are easily verified. It is straightforward to check that the elasticity of the $v$-u ratio generated by the model is exactly $\bar{\varepsilon}_{\theta, S T R O N G}$, where the annuity value of wages is related to the rent parameter $g$ through the formula $g=\frac{r+\delta}{r+\delta+f}(w-b)$. This model is ad hoc in the same way as Hall's rigid wage. In analogy to Hall (2005), one may think of it as an alternative equilibrium selection of a double auction, and interpret it as a norm or social consensus which applies to worker rents rather than the level of wages. An alternative well-known model of wage determination that delivers complete rent rigidity is the Shapiro and Stiglitz (1984) efficiency wage shirking model. ${ }^{10}$

\footnotetext{
${ }^{9}$ The weak bound applies not only if worker rents are Increasing, but also if they are just Procyclical. For the strong bound we can obtain an analogous result, but it requires a stronger version of Condition 2 . If worker rents are Procyclical but not Increasing, then we cannot conclude that $\frac{\partial \mathcal{G}}{\partial p}-\frac{\frac{\partial \mathcal{G}}{\partial p}+\frac{\partial \mathcal{G}}{\partial n} n_{p}}{1-\frac{d n}{d p}} \leq 0$. To insure that $\phi \geq 1$ we need to strengthen $(r+\delta) \frac{\partial \mathcal{J}}{\partial p} \leq \xi$ to $(r+\delta)\left(\frac{\partial \mathcal{J}}{\partial p}+\frac{\partial \mathcal{G}}{\partial p}\right) \leq \xi$. If the model of wage determination satisfies this property along with Condition 1, then the strong bound applies if worker rents are Procyclical.

10 According to Equation (5) in their paper, the worker receives a flow rent $g=\frac{(r+\delta+q) e}{q}$ where $e$ is the cost to the worker of providing effort, and $q$ is the rate at which a shirking worker is caught. Thus rents are completely rigid in response to a shock to aggregate labor productivity as long as both $e$ and $q$ do not vary with productivity $p$.
} 
An interesting question is whether the bound can be attained not only through complete rent rigidity, but also by the models of wage determination under asymmetric information considered in Section 4. There we provide an affirmative answer for the monopoly model.

The model of complete rent rigidity attains the strong bound. The difference between the two bounds is that the first bound neglects the congestion effect. This implies that the weak bound cannot be attained by any model of wage determination that generates a nonzero congestion effect. The congestion effect is positive as long as $\frac{\partial \mathcal{J}}{\partial p}$ is finite. With ex post efficient trade this is always the case. Since the model of complete rent rigidity features ex post efficient trade, it cannot attain the weak bound. Introducing ex post inefficient trade in an ad hoc fashion into the model of complete rent rigidity would enable it to attain the weak bound. ${ }^{11}$

\subsection{Calibration}

We now evaluate the two volatility bounds quantitatively and compare them to Nash bargaining and wage rigidity. To make this comparison we need to compute the elasticities of the $v-u$ ratio associated with these models of wage determination. In analogy to the bounds we express these elasticities in terms of the parameters $\eta, p, b, r, \delta$, together with the equilibrium job finding rate $f$ and the equilibrium annuity value of wages $w$. The formulas are written to facilitate comparison with the bounds:

$$
\varepsilon_{\theta, N A S H}=\frac{p}{p-b}\left(\frac{1}{\frac{1}{1-\eta}}+\frac{w-b}{p-b} \frac{1}{\frac{1}{\eta} \frac{r+\delta+f}{f}}\right)^{-1}, \quad \varepsilon_{\theta, H A L L}=\frac{p}{p-b}\left(\frac{p-w}{p-b} \frac{1}{\frac{1}{1-\eta}}\right)^{-1}
$$

for Nash bargaining and wage rigidity, respectively. ${ }^{12}$ Nash bargaining differs from the strong bound through a higher weight on the term $\frac{1}{1-\eta}$, which stems from the procyclicality of rents. Wage rigidity differs through a zero weight on $\frac{1}{\eta} \frac{r+\delta+f}{f}$, as it completely severs the feedback effect. Our quantitative evaluation is based Shimer's (2005) calibration, which is displayed in Table 1. The model period is a quarter. The interest rate is chosen to match an annual discount factor of 0.953. Shimer infers time series of quarterly separation and job finding

\footnotetext{
${ }^{11}$ Let $\xi(p-n)=e^{\zeta(p-n)}$ be the probability of trade. Set $(r+\delta) J(p, n)=\xi(p-n)(p-n)-g$ if $\xi(p-n)(p-n) \geq g$, setting it to zero otherwise. Then the weak bound is attained for $\zeta \rightarrow \infty$.

${ }^{12}$ In the case of Nash bargaining the annuity value of wages is related to the bargaining parameter through $w=\frac{(r+\delta)[(1-\beta) b+\beta p]+\beta f p}{(r+\delta)+\beta f}$. With wage rigidity the annuity value of wages is simply the rigid wage $w=\bar{w}$.
} 
Table 1

\begin{tabular}{cc}
\hline \hline Parameter & Value \\
\hline$r$ & 0.012 \\
$\delta$ & 0.1 \\
$\eta$ & 0.28 \\
$\frac{b}{\bar{p}}$ & 0.4 \\
$f$ & 1.35 \\
$\beta$ & 0.72 \\
\hline \hline
\end{tabular}

rates from BLS data. He sets $\delta$ equal to the average separation rate of 0.1 . The data on the job finding rate together with data on the $v-u$ ratio is used to estimate the matching function elasticity, yielding $\eta=0.28$. Given the value of the matching function elasticity, Shimer chooses the Nash bargaining parameter $\beta$ such that the Hosios (1990) condition is satisfied, that is $\beta=1-\eta=0.72{ }^{13}$ It is more difficult to calibrate the value of non-market activity $b$. If one is willing to assume that it stems solely from unemployment benefits one can use replacement rates. Proceeding along these lines Shimer sets $\frac{b}{\bar{p}}=0.4$. We return to the calibration of this parameter below.

Notice that the vacancy cost $c$ does not appear in the table. It is pinned down by calibrating the model to match the average job finding rate $f=1.35$.

Shimer's calibration provides all the information needed to evaluate the bounds except for the annuity value of wages (normalized by productivity) $\frac{w}{\bar{p}}$. In his calibration of the model with Nash bargaining Shimer does not need to directly calibrate the annuity value of wages since it is pinned down by choosing the bargaining parameter $\beta$ to satisfy the Hosios condition. The implied value is $\frac{w_{N B}}{p}=0.9826$. When calibrating the model with a rigid wage, Hall (2005) and Shimer (2004) must choose a level of the rigid wage and thus of the annuity value of wages. They sidestep the issue of independently calibrating the wage by adopting the level implied by the model with Nash bargaining under the Hosios condition. We could proceed in the same way. It is desirable, however, to calibrate the wage level independently,

\footnotetext{
${ }^{13}$ Shimer assumes a Cobb-Douglas matching function, for which $\beta=1-\eta$ implies social efficiency of the equilibrium.
} 
using data on hiring costs. To see how this works, notice that Equation (2) can be written as

$$
\frac{c}{q \xi}=\frac{\bar{p}-w}{r+\delta}
$$

The left hand side is the expected cost of hiring a worker, that is the flow cost $c$ times the expected duration of a vacancy $\frac{1}{q \xi}$. Over the life of the match the firm receives the flow $\bar{p}-w$. The present value of this flow must be such that the firm recoups the initial hiring costs. Both Silva and Toledo (2007) as well as Hagedorn and Manovskii (2006) utilize evidence provided by Barron, Berger, and Black (1997) which in turn is based on the 1982 Employment Opportunity Project and the 1992 Small Business Administration survey. This evidence suggests that the average labor cost of hiring a worker corresponds to between 3 and 4.5 percent of the quarterly wage. This does not include costs such as advertisement, and citing further evidence Silva and Toledo (2006) argue that total hiring costs could be as high as 14 percent of the quarterly wage. This is close to the estimate of 13 percent provided by Abowd and Kramarz (2003) for French data. For our purposes it suffices to identify a region in which hiring costs could reasonably lie. Proceeding conservatively based on the evidence above we consider hiring costs between 3 percent and 30 percent of quarterly wages. Using Equation (8) this yields a range $\left[\frac{w_{\min }}{\bar{p}}, \frac{w_{\max }}{\bar{p}}\right]=[0.9675,0.9967]$ for the wage $\frac{w}{\bar{p}}$. Notice that the wage level $\frac{w_{N B}}{\bar{p}}$ implied by the model with Nash bargaining under the Hosios condition is contained in this range and corresponds to hiring costs of 16 percent of quarterly wages.

Figure 3 plots the volatility bounds as a function of the wage $\frac{w}{\bar{p}}$ together with the elasticities implied by wage rigidity and Nash bargaining. The large elasticities generated by wage rigidity make it convenient to use a logarithmic scale. The range $\left[\frac{w_{\min }}{\bar{p}}, \frac{w_{\max }}{\bar{p}}\right]$ based on the evidence on hiring costs is indicated by the shaded area. For US data Shimer finds that the relative volatility of the $v$ - $u$ ratio and labor productivity (as measured by the coefficient of variation) is $\frac{\sigma_{\theta}}{\sigma_{p}}=19$, and this level is indicated by a horizontal line as a point of reference. The downward sloping dashed line represents Nash bargaining. The wage $\frac{w_{N B}}{\bar{p}}$ associated with the bargaining share $\beta=0.72$ implies an elasticity of 1.72 . This is an order of magnitude smaller than the relative volatility of the $v$ - $u$ ratio and productivity in the data, which is precisely the failure of the model emphasized by Shimer (2005). The upward sloping dashed-dotted line represents the elasticity generated by wage rigidity. The range of elasticities induced by a rigid wage in the shaded region is $[42.74,420.88]$. Thus for reasonable values of hiring costs a rigid wage 
Figure 3: Volatility Bounds

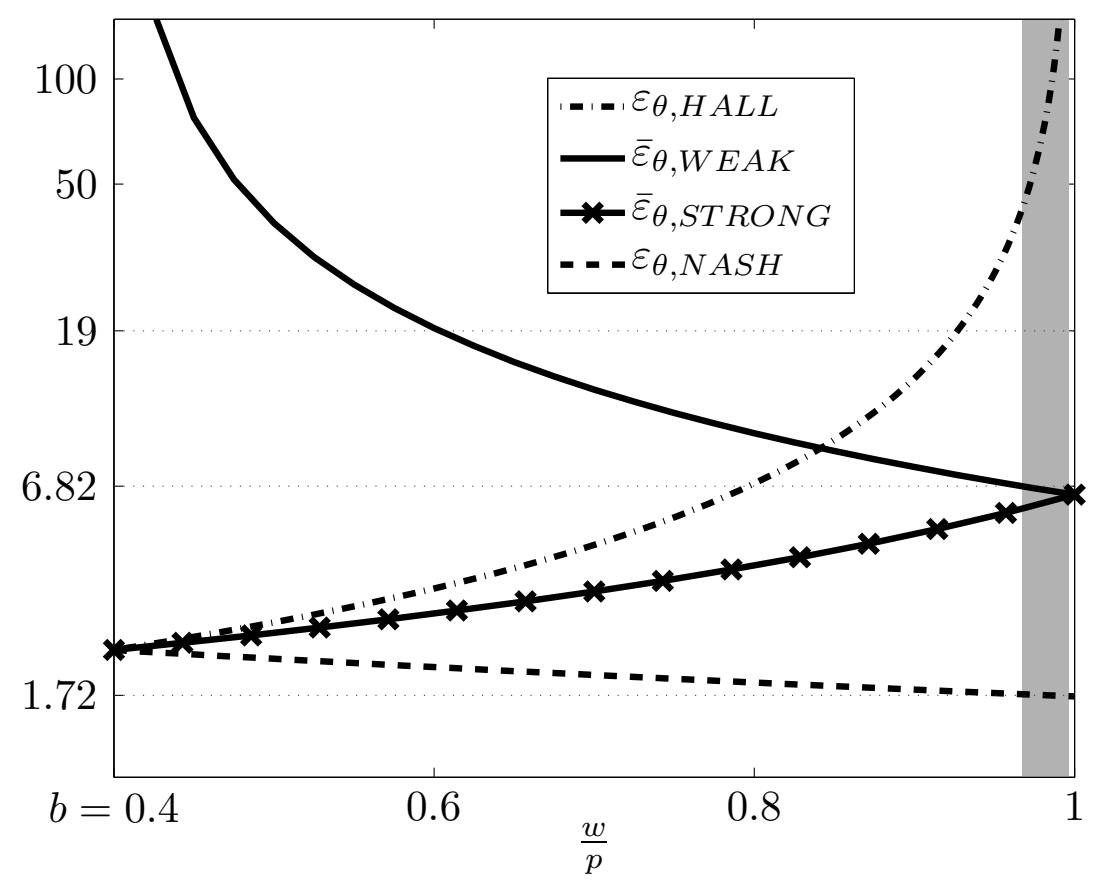

generates an elasticity $\varepsilon_{\theta}$ in excess of the empirical relative volatility $\frac{\sigma_{\theta}}{\sigma_{p}}$. This suggests that wage rigidity generates more than enough amplification, leaving room for the possibility that a weaker form of rigidity could suffice to match observed labor market volatility. ${ }^{14}$ In particular, one may ask whether rent rigidity is capable of doing so.

A negative answer to this question is provided by the plots of the two volatility bounds. Both are represented by solid lines, the strong bound with crosses. Over the shaded region of interest the two bounds are quantitatively close and vary little: the weak bound ranges from 6.48 to 6.82 whereas the strong bound ranges from 5.88 to 6.38 . Thus for reasonable values of hiring costs, models of wage determination that generate procyclical worker rents can generate at most a third of observed volatility. ${ }^{15}$ In other words, replacing Nash bargaining by a model

\footnotetext{
14 The range of elasticities $[42.74,420.88]$ overstates the case that a rigid wage generates more than enough amplification. As discussed before, the case of permanent shocks provides an upper bound for the case in which persistence is calibrated to US data. To investigate this issue we calibrated the rigid wage model following the approach of Shimer (2005) for wage levels in the shaded range and obtained the range [20.8, 57.9] for the relative coefficient of variation $\frac{\sigma_{\theta}}{\sigma_{p}} \frac{\mu_{p}}{\mu_{\theta}}$. Thus it is still the case that a rigid wage generates relative volatility in excess of what is observed.

15 Mortensen and Nagypal (2005) argue that 7.52 is a more appropriate target for the elasticity $\varepsilon_{\theta}$ than the value of 19 implicit in Shimer (2005). Their argument is based on the relatively low empirical correlation
} 
with rigid rents does not provide a silver bullet for resolving the inability of the model to match observed labor market fluctuations. Nevertheless, some amplification vis-a-vis Nash bargaining is not ruled out by the bound. Thus it is of interest whether the bound can be attained. We have already shown that the strong bound can be attained with complete rent rigidity, and in Section 4 we show how it is attained by a model of wage determination under asymmetric information. ${ }^{16}$

Notice that the two bounds coincide for $\frac{w}{\bar{p}}=1$. Recall that the difference between the two bounds is that the weak bound neglects the congestion effect. This effect is zero for $\frac{w}{\bar{p}}=1$. If the model is calibrated to a lower annuity value of wages $\frac{w}{\bar{p}}<1$, then a gap appears between the two bounds. The weak bound increases as it only reflects the feedback effect, whose strength is reduced if wages and thus worker rents are small. It asymptotes to infinity because the feedback effect disappears as the annuity value of wages approaches the value of non-market activity $b$. In contrast, the strong bound is an increasing function of the annuity value of wages. Recall from Equation (7) that the strong bound is a weighted average of the feedback and the congestion effect. The weight on the feedback effect is an increasing function of the annuity value of wages. Thus the slope of the strong bound depends on which of the two effects puts a tighter limit on labor market fluctuations. The plot shows that the congestion effect is more severe: calibrating the model to match a lower annuity value of wages reduces the amplification generated by rent rigidity. Setting $w=b$ puts all weight on the congestion effect and yields $\bar{\varepsilon}_{\theta, S T R O N G}=\frac{\bar{p}}{\bar{p}-b} \frac{1}{1-\eta}=2.31$; putting all weight on the feedback effect by setting $w=\bar{p}$ yields $\bar{\varepsilon}_{\theta, S T R O N G}=\frac{\bar{p}}{\bar{p}-b} \frac{1}{\eta} \frac{r+\delta+f}{f}=6.45$. Importantly, the strong bound is finite between labor market tightness and labor productivity, suggesting that part of fluctuations in labor market tightness is due to other shocks. Rent rigidity comes close to attaining their 7.52 target. Thus while rent rigidity alone cannot enable the model solely driven by productivity shocks to match observed volatility, a model with rigid rents and multiple shocks could get close. On the other hand, the appropriate target for $\varepsilon_{\theta}$ could be even higher than Shimer's 19 if the low empirical correlation between labor market tightness and labor productivity reflects measurement error in the latter rather than other shocks.

${ }^{16}$ That the model can attain the bound does not mean that the relative coefficient of variation $\frac{\sigma_{\theta}}{\sigma_{p}} \frac{\mu_{p}}{\mu_{\theta}}$ from simulations with productivity persistence calibrated to US data is necessarily close to the strong bound, for the reason already discussed in Footnote 14. Simulating the model for the usual range of wages, following the approach of Shimer (2005), yields yields the range $[5.18,6.30]$ for the relative coefficient of variation, compared to the corresponding values for the bound $[5.88,6.38]$. Thus the simulated model comes very close to attaining bound. 
irrespective of the annuity value of wages. In this sense it requires less empirical information than the weak bound. In particular, using only parameters from Shimer's calibration and without calibrating hiring costs we can conclude that models of wage determination subject to the strong bound cannot generate more than a third of observed volatility.

The congestion effect is maximized if the annuity value of wages $w$ equals the value of non-market activity. In this case Nash bargaining, rent rigidity and wage rigidity all coincide. The plot of the strong bound illustrates that rent rigidity lies between Nash bargaining and wage rigidity, but quantitatively it is much closer to the former (remember that the scale is logarithmic).

Why does the congestion effect put a tighter limit on fluctuations than the feedback effect in Shimer's calibration? The size of the feedback effect is determined by the term $\frac{1}{\eta} \frac{r+\delta+f}{f}$. Since $f \gg r+\delta$ this is well approximated by $\frac{1}{\eta}$. The strength of the congestion effect is determined by $\frac{1}{1-\eta}$. Thus which one of the two effects puts a tighter bound on amplification in essence just depends on the matching function elasticity $\eta$. If $\eta$ is below $\frac{1}{2}$ as in Shimer's calibration, then an increase in the number of vacancies primarily causes congestion with little effect on the job finding rate and a correspondingly weak feedback effect. If instead $\eta$ is above $\frac{1}{2}$ an increase in vacancies is associated with a strong response of the number of matches, causing little congestion while substantially increasing the job finding rate of unemployed workers. Since the elasticity $\eta$ plays such an important role it is useful to consider how changing this parameter affects the bounds. This is particularly important since Shimer's value lies at the low end of the reasonable range of $[0.3,0.5]$ identified in the survey by Petrongolo and Pissarides (2001). In Figure 4 we consider the other end of the spectrum $\eta=0.5$. The strong bound is flat as the congestion and feedback effects are now roughly balanced. The feedback effect is strengthened relative to Shimer's calibration, and now generates a much tighter bound over the shaded region of about 3.61. This is not much higher than the maximal elasticity of 3.33 which Nash bargaining is able to generate, albeit only for very large values of hiring costs.

Next we discuss the role of the value of non-market activity $b$. Our two bounds and the elasticities of the $v$ - $u$ ratio generated by Nash bargaining and wage rigidity have one thing in common: they all include the term $\frac{\bar{p}}{\bar{p}-b}$. To understand the role of this term it is useful to think of the gains from market activity $\bar{p}-b$ as the driving force of the model. Then $\frac{\bar{p}}{\bar{p}-b}$ is the 
Figure 4: Volatility Bounds $\eta=0.5$

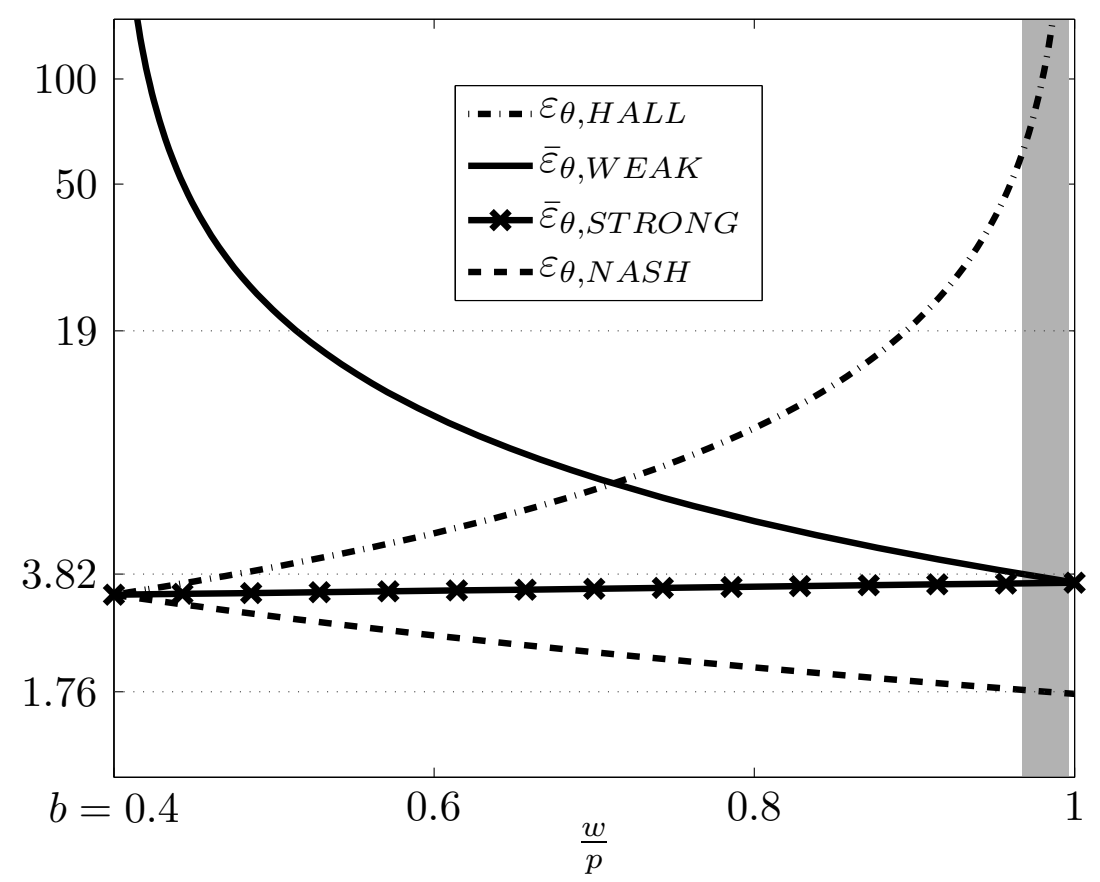

elasticity of this driving force with respect to productivity. If $b$ is close to $\bar{p}$, then even small fluctuations in productivity can generate large fluctuations in the driving force of the model. Thus even if the model does not strongly amplify fluctuations in $\bar{p}-b$ it could still generate large labor market fluctuations for sufficiently large $b$. In Shimer's calibration $\frac{\bar{p}}{\bar{p}-b}=1.67$ is small. Hagedorn and Manovskii (2006) show that with $\frac{b}{\bar{p}}=0.955$ even Nash bargaining can match observed labor market fluctuations. Analogously one may ask: at what value of non-market activity can the model of complete rent rigidity match observed volatility? For an annuity value of wages $\frac{w_{N B}}{\bar{p}}=0.9826$ the answer is $\frac{b}{\bar{p}}=0.828$.

\section{Wage Determination under Asymmetric Information}

We now consider classic models of bargaining with incomplete information, that have been studied very extensively. We want to check whether these models exhibit the properties, such as Increasing worker rents, under which the labor market volatility bounds apply. These are comparative statics properties of equilibrium outcomes of the bargaining game. Comparative statics are meaningful only when the equilibrium prediction is unique. Thus, we focus our attention on bargaining protocols for which the literature has established outcome uniqueness, 
possibly subject to appropriate refinements: one-sided offers with and without commitment to the offer, strategic bargaining with one-sided asymmetric information, and the constrained efficient allocation maximizing expected gains from trade. In each case, the comparative statics properties of the unique equilibrium outcome are unknown, so we have to derive them anew.

There is an additional reason to focus on unique outcomes. If the bargaining game has multiple outcomes, then this could be exploited to generate amplification by selecting an outcome favorable to firms in booms and an outcome favorable to workers in recessions. However, asymmetric information is not needed to do this. In fact, Hall (2005) exploits multiplicity in this way to provide a foundation for wage rigidity in the model with symmetric information. He assumes that the wage is set through a double auction. The key feature of the double auction under symmetric information environment is that any split of the gains from trade is an equilibrium. Thus one can select the rigid wage as the equilibrium for all combinations of $p$ and $n$, as long as this wage is always in the bargaining set $[n, p]$. Since multiplicity can be used to generate the observed amount of volatility even under symmetric information, the only interesting question is whether models with asymmetric information are capable of doing so without exploiting multiplicity. Hence our focus on bargaining protocols with unique outcomes.

In our setting, private information concerns the idiosyncratic productivity component of the match, $y$, and amenity value for the worker, $z$. The distributions $F_{Y}$ and $F_{Z}$ have support $[\underline{y}, \bar{y}]$ and $[\underline{z}, \bar{z}]$, respectively, with $\underline{y}, \underline{z}, \bar{y}, \bar{z} \in \overline{\mathbb{R}}$. We now introduce a standard technical assumption about private information.

Assumption 1. (Monotone Virtual Valuations) The "virtual valuations" $y-\frac{1-F_{Y}(y)}{F_{Y}^{\prime}(y)}$ and $z-\frac{1-F_{Z}(z)}{F_{Z}^{\prime}(z)}$ are strictly increasing and continuously differentiable on $[\underline{y}, \bar{y}]$ and $[\underline{z}, \bar{z}]$, respectively.

For each of the rent-sharing models that we examine, we proceed as follows: we show that Condition 1 (c) holds, and then that expected rents are a function of $p$ and $n$ only through their difference $p-n$, with $\mathcal{G}^{\prime}(p-n) \geq 0$ and $\mathcal{J}^{\prime}(p-n) \geq 0$. This is all that we need for the first bound to apply. In fact, it implies that

$$
\frac{\partial \mathcal{G}}{\partial p}=\mathcal{G}^{\prime}(p-n)=-\frac{\partial \mathcal{G}}{\partial n} \geq 0
$$


which implies Increasing worker rents and thereby Condition 1 (a). It also implies

$$
-\frac{\partial \mathcal{J}}{\partial n}=\mathcal{J}^{\prime}(p-n) \geq 0
$$

which is Condition 1 (b). For the second bound, we also verify Condition 2 directly.

\subsection{Monopoly}

First, we consider the simplest game in which the privately uninformed party makes a takeit-or-leave-it offer to the informed party. This is the model suggested by Shimer (2005) in his conjecture that asymmetric information may generate amplification. In this model, the relationship ends if the informed party rejects the offer. In other words, the uninformed party can commit not to make another offer if its first offer is rejected. This game has a unique equilibrium, which is constrained ex ante efficient in the sense that the offer-making party's welfare cannot be improved further given information asymmetry (Satterthwaite and Williams (1989)). Yet, it does not maximize ex ante gains from trade, due to the monopoly distortion. We analyze separately the two cases of unilateral wage offer by the firm and wage request by the worker, because the properties used to derive the second bound are not symmetric for firms and workers.

Take-It-or-Leave-It Wage Offer by the Firm. A firm of type $y$ offers a wage $w_{M}$ to the job applicant, who is then indifferent between accepting and rejecting it to stay unemployed when his amenity value is exactly $z_{M}=n-w_{M}$. If $z \geq z_{M}$, and the offer is accepted, an event with chance $1-F_{Z}\left(z_{M}\right)$, the firm earns flow profits $p+y-w_{M}=p-n+y+z_{M}$. Equivalently, the firm chooses the threshold $z_{M}$, rather than the wage $w_{M}$, to maximize the PDV of:

$$
\left[1-F_{Z}\left(z_{M}\right)\right]\left(p-n+y+z_{M}\right)
$$

The well-known first order condition is

$$
p-n+y+z_{M}=\frac{1-F_{Z}\left(z_{M}\right)}{F_{Z}^{\prime}\left(z_{M}\right)} .
$$

The left hand side is the gain from trading with an additional worker. However, if the firm wants to trade with more workers, it has to pay higher informational rents to the workers (types, values of $z$ ) it is already trading with. The right hand side gives the number of workers that receive higher rents relative to the number of workers gained from reducing $z_{M}$. 
If (10) has an interior solution $z_{M}(p-n+y)$, by Assumption 1, this is unique, differentiable, and the global maximizer. Assumption 1 allows for finite lower and upper bounds, so $z_{M}(p-$ $n+y)$ could be at a corner, equal to the lower bound $\underline{z}$ (the offer is accepted for sure) if $p-n+y+\underline{z} \geq\left[F_{Z}^{\prime}(\underline{z})\right]^{-1}$, that is if the gain from trading with more workers always outweighs the cost of higher informational rents, and equal to the upper bound $\bar{z}$ (the offer is rejected for sure) if $p-n+y+\bar{z} \leq 0$. One may expect that corner solutions may generate sufficient wage rigidity to escape the bounds. We will show that this is not the case.

From acceptance of its offer, the firm learns that the type of the worker is at least $z_{M}(p-$ $n+y)$. Although it has obtained this information, the firm would not want to revise its wage offer if it were allowed to do so. ${ }^{17}$ Thus the key assumption regarding commitment is not that the firm can commit not to make a lower wage offer after acceptance, but that it can commit not to make a higher wage offer after being rejected.

It is now straightforward to map this model of wage determination into the notation of Section 2, and check that it satisfies the properties there introduced:

$$
\begin{aligned}
G(y, z, p, n) & =x(y, z, p-n) \frac{z-z_{M}(p-n+y)}{r+\delta}, \\
J(y, z, p, n) & =x(y, z, p-n) \frac{p-n+y+z_{M}(p-n+y)}{r+\delta}, \\
x(y, z, p-n) & =\mathbb{I}\left\{z \geq z_{M}(p-n+y)\right\} .
\end{aligned}
$$

The trade decision $x$ depends on $p$ and $n$ only through their difference $p-n$. Moreover, the same is true for the functions $G$ and $J$ and for their unconditional counterparts, that we can write as $\xi(p-n), \mathcal{G}(p-n)$ and $\mathcal{J}(p-n)$ from now on. Inspecting the firm's objective in (9), an increase in $p-n+y$ raises the marginal gain from trade by lowering the threshold $z_{M}$. By a monotone comparative statics argument, or by the implicit function theorem, $z_{M}(p-n+y)$ is weakly decreasing (and strictly so over the range where the solution is interior). Consulting Equation (11), this implies that $x(y, z, p-n)$ is non-decreasing in both $y$ and $z$, and Condition 1 (c) is verified. As discussed earlier, for the first bound we just need $\mathcal{G}^{\prime}(p-n), \mathcal{J}^{\prime}(p-n) \geq 0$.

Define the worker's average gains from trading with a firm of type $y$ :

$$
(r+\delta) \mathcal{G}(p-n \mid y) \equiv \int_{z_{M}(p-n+y)}^{\bar{z}}\left[z-z_{M}(p-n+y)\right] d F_{Z}(z)
$$

\footnotetext{
${ }^{17}$ Formally, the objective of the firm facing the distribution of worker types truncated at $z_{M}(p-n+y)$ is given by $p-n+y+z_{M}$ for $z_{M} \leq z_{M}(p-n+y)$ and $\frac{1-F_{Z}\left(z_{M}\right)}{1-F_{Z}\left(z_{M}(p-n+y)\right)}\left(p-n+y+z_{M}\right)$ for $z_{M} \geq z_{M}(p-n+y)$. The unique maximizer is still $z_{M}(p-n+y)$.
} 
This function is differentiable everywhere, including at the two threshold values of $y$ where the first order condition (10) holds with equality for the corners $\underline{z}$ and $\bar{z}$, with

$$
(r+\delta) \mathcal{G}^{\prime}(p-n \mid y)=-z_{M}^{\prime}(p-n+y)\left[F_{Z}\left(z_{M}(p-n+y)\right)\right] \geq 0 .
$$

The firm expands the range of workers it is trading with by $-z_{M}^{\prime}(p-n+y)$, so the informational rents of all worker types that it is already trading with have to increase by exactly this amount. If $z_{M}(p-n+y)$ is at a corner $\underline{z}$ or $\bar{z}$, clearly $z_{M}^{\prime}(p-n+y)=0$ and the inequality still applies. By definition $\mathcal{G}(p-n)=\int \mathcal{G}(p-n \mid y) d F_{Y}(y)$, so that

$$
\mathcal{G}^{\prime}(p-n)=\int \mathcal{G}^{\prime}(p-n \mid y) d F_{Y}(y) \geq 0
$$

For the firm, the maximized value for type $y$ is

$$
(r+\delta) \mathcal{J}(p-n \mid y)=\left[1-F_{Z}\left(z_{M}(p-n+y)\right)\left[p-n+y+z_{M}(p-n+y)\right]\right.
$$

Differentiation yields

$$
(r+\delta) \mathcal{J}^{\prime}(p-n \mid y)=1-F_{Z}\left(z_{M}(p-n+y)\right)
$$

If the firm is at a corner this follows immediately, as $z_{M}(p-n+y)$ does not respond to a change in $p-n$. If the solution to the firm's problem is interior, this relationship follows from the envelope theorem. Since the threshold $z_{M}$ is chosen optimally, the firm cannot gain at the margin from adjusting the threshold, so the benefit from an increase in $p-n$ is just the direct effect on the rents that the firm earns from the workers that it is already trading with. It follows that $\mathcal{J}(p-n \mid y)$ is continuously differentiable, and differentiation under the integral sign yields

$$
\begin{aligned}
(r+\delta) \mathcal{J}^{\prime}(p-n) & =\int(r+\delta) \mathcal{J}^{\prime}(p-n \mid y) d F_{Y}(y) \\
& =\int\left[1-F_{Z}\left(z_{M}(p-n+y)\right)\right] d F_{Y}(y)=\xi(p-n) \geq 0
\end{aligned}
$$

This also establishes that Condition 2 holds with equality. Recall our discussion that Condition 2 is innocuous if trade is ex post efficient, because in this case the envelope theorem implies $(r+\delta)\left(\frac{\partial \mathcal{G}}{\partial p}+\frac{\partial \mathcal{J}}{\partial p}\right)=\xi$. Here Condition 2 again follows from the envelope theorem which delivers $(r+\delta) \frac{\partial \mathcal{J}}{\partial p}=\xi$. At the same time, due to the monopoly inefficiency, trade is generally not ex post efficient $(r+\delta)\left(\frac{\partial \mathcal{G}}{\partial p}+\frac{\partial \mathcal{J}}{\partial p}\right)>\xi$.

We summarize these results in the following: 
Proposition 3. Under Assumption 1 the model of wage determination through a take-itor-leave-it offer by the firm to a privately informed worker satisfies Conditions 1 and 2 and Increasing worker rents. Thus, both labor market volatility bounds of Propositions 1 and 2 apply.

Take-It-or-Leave-It Wage Request by the Worker. By symmetry with the case that we just analyzed, when the worker makes a take-it-or-leave-it wage request from the firm, Condition 1 is satisfied and worker rents are Increasing. These are all the properties needed to apply the weak bound of Proposition 1.

To apply the strong bound, we cannot invoke symmetry with the previous case: there, we only established that the rents of the offer-making party satisfy Condition 2, while now the firm is at the receiving end of the offer. Using notation symmetric to the firm offer model, in the worker request model

$$
(r+\delta) \mathcal{J}^{\prime}(p-n \mid z)=-y_{M}^{\prime}(p-n+z)\left[1-F_{Y}\left(y_{M}(p-n+z)\right)\right]
$$

at points of differentiability of $y_{M}(p-n+z)$, the threshold productivity level chosen by the worker with amenity value $z$. Only firm types that the worker has already been trading with experience an increase in their informational rent, which is why the probability of trade $1-F_{Y}\left(y_{M}(p-n+z)\right)$ appears in Equation (12). How large the increase in the informational rent is for these firm types depends on how many more firm types the worker wants to trade with, that is, on the drop in the threshold $-y_{M}^{\prime}(p-n+z)$. If the worker reduces the request and thus the feasible productivity threshold substantially, then the firm will experience a large increase in its informational rents. Now suppose that the worker reduces the threshold less than one for one with an increase in $p-n$, that is $-y_{M}^{\prime}(p-n+z) \leq 1$. Then

$$
\begin{aligned}
(r+\delta) \mathcal{J}^{\prime}(p-n) & =\int-y_{M}^{\prime}(p-n+z)\left[1-F_{Y}\left(y_{M}(p-n+z)\right)\right] d F_{Z}(z) \\
& \leq \int\left[1-F_{Y}\left(y_{M}(p-n+z)\right)\right] d F_{Z}(z)=\xi(p-n),
\end{aligned}
$$

enough for Condition 2. The following strengthening of the second part of Assumption 1 suffices for $-y_{M}^{\prime} \leq 1$.

Assumption 2. (Monotone Hazard Rate) The hazard rate $\frac{F_{Y}^{\prime}(y)}{1-F_{Y}(y)}$ is weakly increasing and continuously differentiable on $[\underline{y}, \bar{y}]$. 
To understand the role of a monotone hazard rate, consult the worker's first order condition for an optimal wage request to the firm:

$$
p-n+y_{M}+z=\frac{1-F_{Y}\left(y_{M}\right)}{F_{Y}^{\prime}\left(y_{M}\right)} .
$$

If in response to an increase in $p-n$ the worker reduced $y_{M}$ one for one, then the left hand side, which is the marginal benefit from trading with another firm type, would be unchanged. However, under Assumption 2 the worker would end up at a point with a lower hazard rate, that is the loss of trade associated with a more aggressive wage request would be smaller relative to the number of firms that would pay the higher wage. It follows that it is optimal to reduce the threshold less than one for one.

Thus we obtain the following:

Proposition 4. Under Assumption 1 the model of wage determination through a take-it-orleave-it request by the worker to a privately informed firm satisfies Condition 1 and Increasing worker rents. Thus, the weak labor market volatility bound of Proposition 1 applies. If Assumption 1 is strengthened to Assumption 2, then this model also satisfies Condition 2 and the strong volatility bound of Proposition 2 also applies.

Kennan (2006) also considers the model with take-it-or-leave-it offers, and he is able to generate large fluctuations in unemployment and vacancies. As discussed in the introduction, his approach exploits asymmetric information in a different way from what we consider in this paper. We ask how the presence of asymmetric information changes the response of the labor market to an increase in aggregate labor productivity, but the extent of private information itself is acyclical. In contrast, in Kennan (2006) exogenous fluctuations in the shape of the distribution of types are the key driving force of labor market fluctuations. Specifically, he assumes that the match specific productivity component can be either high or low, and that the high value is more likely in booms. He assumes that parameters are such that pooling occurs in both booms and recessions: workers always request the wage that makes the low firm type indifferent. Since the value of unemployment is procyclical, it follows that worker rents are countercyclical. ${ }^{18}$

\footnotetext{
18 Translated into our notation, in Kennan (2006) both $p$ and $F_{Y}$ vary over the cycle. It is straightforward to decompose labor market volatility into three sources: $p, F_{Y}$, and an interaction term. In his calibration
} 
In Section 3.2 we demonstrated that the strong labor market volatility bound can be attained by the model of complete rent rigidity. We now show that there exist beliefs such that the monopoly model also attains the bound.

Attaining the Strong Bound in Firm Offer Monopoly. The idea is to construct beliefs such that the monopoly model maps exactly into the model of complete rent rigidity, that is the worker receives a fixed flow rent $g>0$. For simplicity there is no heterogeneity on the firm side, that is $F_{Y}$ is degenerate. Let $w=b+\frac{r+\delta+f}{r+\delta} g$. As in the model with complete rent rigidity, this turns out to be the equilibrium annuity value of wages. Let $j=p-w=p-b-\frac{r+\delta+f}{r+\delta} g$, which will be the equilibrium value of profits. Consider beliefs

$$
F_{Z}(z) \equiv \frac{1-\frac{j}{z+g+j}}{1-\frac{j}{\bar{z}+g+j}}
$$

with support $[\underline{z}, \bar{z}]$ where the lower bound is $\underline{z}=-g$ and the upper bound is chosen to insure a zero mean, that is it is the unique positive solution to the equation $\int_{\underline{z}}^{\bar{z}} z F_{Z}(d z)=0$. We will show that in steady state the firm trades with all worker types. Steady state flow rents are $p-n=g+j$. More importantly, we show that trading with all worker types remains optimal in response to a productivity shock, which in turn implies $\mathcal{G}^{\prime}(g+j)=0$. Thus this example maps exactly into the model of complete rent rigidity.

First note that if the firm chooses to trade with all worker types, then flow worker rents are

$$
(r+\delta) \int_{\underline{z}}^{\bar{z}}(z-\bar{z}) d F_{Z}(z)=-\underline{z}=g .
$$

Furthermore, if flow worker rents are $g$, then equilibrium condition (3) implies that in equilibrium $p-n=g+j$. Thus it remains to show that it is indeed optimal for the firm to choose the lower bound $\underline{z}$ for a neighborhood around $p-n=g+j$. Evaluating the objective of the firm at $p-n=g+j$ yields

$$
\left(1-F_{Z}(z)\right)(p-n+z)=\left(1-F_{Z}(z)\right)(z+g+j)=\frac{j}{1-\frac{j}{\bar{z}+g+j}}\left(1-\frac{z+g+j}{\bar{z}+g+j}\right),
$$

a strictly decreasing function of $z$.

While monopoly can attain the bound for some beliefs, this is not to say that it generically leads to amplification vis-a-vis Nash bargaining. It is easy to construct beliefs such that the fluctuations in $F_{Y}$ alone generate about 85 percent of observed volatility in the $v$ - $u$ ratio, while the contribution of $p$ and of the interaction term are small. 
response of the labor market to a productivity shock is muted. Indeed, in his conjecture Shimer (2005) merely argues that the model breaks the direct link between labor productivity and the $v-u$ ratio by making the response to a productivity shock a function of the hazard rate. Thus at least the model adds may degrees of freedom that one may expect should enable the model to match observed volatility. Our results imply that this is not the case: the degrees of freedom are not enough to generate amplification in excess of the strong labor market volatility bound. Moreover, if one chooses the distribution of beliefs to maximize amplification, one obtains precisely complete rent rigidity.

\subsection{Strategic Bargaining with One-Sided Private Information}

We now move away from the case of extreme commitment to a (take-it-or-leave-it) offer, and towards repeated, strategic bargaining. The theory of sequential non-cooperative bargaining with private information has produced compelling predictions only for the case of one-sided asymmetric information, that we address first. In the two-sided case, the wealth of Bayesian equilibria makes alternating-offer games problematic as tools of analysis, despite the realism of the setup. In that case, we will consider the unique allocation that maximizes expected gains from trade through a mediator.

We focus on some popular and natural extensive forms of the bargaining game. In the previous subsection we analyzed the case of a unilateral offer with commitment to a privately informed party. In that case, the private information of the offer-making party is irrelevant, so that solution applies both to one-sided and two-sided asymmetric information. We showed that our bounds apply. Therefore, we now assume that parties cannot commit to walk away when the opponent declines its offer, but they can commit to their offer should it be accepted.

The only change in the environment is that information is private only on one side. To fix ideas, assume that the firm is uninformed about $z$ but the worker observes $y$. The parties then enter a bargaining phase. The firm (uninformed party) makes the first wage offer $w$. If this is accepted, from then until the (exogenous) end of the match the firm has flow profits $p+y-w$, the worker has flow payoffs $w+z-n$, the total surplus is $p+y+z-n$. If negotiations end unsuccessfully, the firm obtains zero and the worker obtains a flow value $n$. Fix numbers

$\beta \in[0,1], \rho_{\text {work }} \geq 0, \rho_{\text {firm }} \geq 0$. If the first offer is declined, negotiations break down with 
chance $1-e^{-(1-\beta) \Delta}$, continue with chance $e^{-(1-\beta) \Delta}$ and, after a delay $\Delta$ and with discounting cost $e^{-\rho_{\text {firm }} \Delta}$, the worker makes a new wage request. Again, if this is declined by the firm, negotiations break down with chance $1-e^{-\beta \Delta}$ and continue with complementary probability, in which case payoffs are further discounted at rate $e^{-\rho_{\text {work }} \Delta}$. Notice then that the discount rate after a firm offer is declined is effectively equal to $1-\beta+\rho_{\text {firm }}$, and after a worker offer is declined it equals $\beta+\rho_{\text {work }}$. We study Perfect Bayesian Nash Equilibria (PBE) when $\Delta \rightarrow 0$.

This model nests several well-known models as special cases. First, let the uninformed party $i \in\{$ work, firm $\}$ make the first offer. If the discount rate $\rho_{i}$ that applies after the first offer is infinite, we are back to the previous case of monopoly with commitment. Therefore, from now on we assume $\rho_{i}<\infty$. We analyze first the simpler case where repeated offers are effectively one-sided.

Coase Conjecture. Suppose again for illustration that the firm is uninformed and $\beta=$ $\rho_{\text {work }}=0 \leq \rho_{\text {firm }}<\infty$. In this case, any request by the worker can be rejected at no cost by the firm, because negotiations continue for sure and there is no discounting after a worker's request is declined, but declining a firm's offer implies an efficiency loss $(1-\beta>0)$. Thus, offers are effectively one-sided. This is the case of a monopolist (firm) selling a durable good (job) to a buyer (worker) who has private information about his own valuation $(z)$ for the job. The firm (uninformed) makes the first offer. As is well-known, the unique PBE as $\Delta \rightarrow 0$ dictates that negotiations end immediately and the worker extracts all rents from the firm, the Coase Conjecture (Gul and Wilson (1986)). We now examine the properties of this model of wage determination. We have two subcases.

In the Gap case the firm's draw of match productivity $y$ is such that there are gains from trade for sure, $p+y+\underline{z}-n>0$. Then the sequential equilibrium wage of the subgame following the observation of $y$ is $w(p, n, y)=n-\underline{z}<p+y$, all worker types accept if payoffs are $w+z-n=z-\underline{z}>0$, the firm makes profits $p+y-w=p-n+y+\underline{z}>0$. In the No-Gap case, given productivity $y$, gains from trade exist only for some worker types $z$, such that $p+y+\underline{z}-n \leq 0<p+y+\bar{z}-n$. Then, the unique equilibrium satisfying a stationarity condition on strategies has the following outcome. The wage offer $w(p, n, y)=p+y$ is accepted immediately, the firm is left with nothing, workers with $z \geq n-(p+y)$ accept and obtain rents $p+y+z-n$, the other worker types decline. 
Trade is ex post efficient, so the ex ante probability of trade is simply $\xi(p-n)=\operatorname{Pr}(p-$ $n+y+z \geq 0$ ) and Condition 1 (c) is verified. Notice that once again not only the trade decision but also the split of rents depends on $p$ and $n$ only through their difference $p-n$. We are left to verify $\mathcal{G}^{\prime}, \mathcal{J}^{\prime} \geq 0$. The firm's rents are

$$
(r+\delta) \mathcal{J}(p-n)=\mathbb{E}_{y, z}[p+y-w(p, n, y)]=\int_{n-p-\underline{z}}^{\bar{y}}(p-n+y+\underline{z}) d F_{Y}(y)
$$

with

$$
(r+\delta) \mathcal{J}^{\prime}(p-n)=1-F_{Y}(n-p-\underline{z}) \in[0, \xi(p-n)]
$$

implying Conditions 1 (b) and 2. The fact that trade is ex post efficient implies that $(r+$ $\delta)\left(\mathcal{G}^{\prime}+\mathcal{J}^{\prime}\right)=\xi$, so it immediately follows that $(r+\delta) \mathcal{G}^{\prime} \in[0, \xi]$. Therefore, the worker and firm informational rents have symmetric properties, and we can swap their roles. These facts establish:

Proposition 5. Under Assumption 1 the "Coase Conjecture" model of wage determination through quickly repeated unilateral offers by the uninformed party in stationary strategies satisfies Conditions 1 and 2 and Increasing worker rents. Thus, the labor market volatility bounds of Propositions 1 and 2 apply.

Menzio (2005b) proposes a model of wage determination that delivers the same implications as the Coase conjecture, albeit through a different mechanism. In his model workers are uninformed. As under the Coase conjecture, the wage requested by the worker in equilibrium is given by the productivity of the lowest firm type: $w=p+\underline{y}$. The mechanism is as follows. The firm employs many workers. If the firm grants a wage in excess of $p+\underline{y}$ to one worker, then all its other employees learn that the firm's type is higher than $\underline{y}$, and they successfully request wage increases. This spillover of information enables the firm to reject wage requests in excess of $p+\underline{y}$. As the implied wage is the same as under the Coase conjecture, it follows that the labor market volatility bounds apply to this model of wage determination as well, as long as private information is acyclical as it is assumed to be here. Menzio uses this model to generate rigid wages as follows. He assumes that the lower bound of the distribution of firm productivity is acyclical. Translated into our notation, $p+\underline{y}$ remains constant over the cycle. As aggregate labor productivity is higher in booms while the lower bound is acyclical, it must be that the shape of the distribution $F_{Y}$ is changing over the cycle. Thus Menzio's approach 
is similar to Kennan (2006) in the sense that it relies on exogenous fluctuations in the shape of the distribution of types in order to generate large labor market fluctuations.

Alternating Offers. Now suppose that $\beta \in(0,1)$, so declining an offer can always lead to a breakdown in negotiations, and $\rho_{\text {firm }}=\rho_{\text {work }}=r \geq 0$. These assumptions provide some physical commitment to parties to walk away after receiving a negative answer to an offer, and enhance the bargaining power of the uninformed party. We illustrate equilibrium predictions drawing from Grossman and Perry (1986), Gul and Sonnenschein (1988), and Menzio (2005a).

In this game, Stationarity of equilibrium strategies is not sufficient to pin down a unique PBE, and one needs to impose also a Monotonicity requirement on beliefs. Then, the unique $\mathrm{PBE}$ is similar to the Coase conjecture, with the only twist that $\beta$ measures the bargaining power of the worker. That is, suppose now (for the sake of variety) that the firm is privately informed about $y$, while $z$ is common knowledge and the worker of type $z$ makes the first wage request. Then, in the Gap case, $p+\underline{y}+z-n>0$, the unique PBE wage first requested by the worker of type $z$ (and accepted by all firms) is $w(p, n, z)=\beta(p+\underline{y})+(1-\beta)(n-z)$ and the worker obtains payoff $z-n+w=\beta(p+\underline{y}+z-n)$. That is, the wage must reflect the worker's valuation because with some probability (decreasing in $\beta$ ): negotiations break down, the firm does not get its turn to counteroffer and it loses everything. This makes the firm more willing to accept a high wage requests, the more so the higher $\beta$. The firm earns $p+y-w(p, n, z)=y-\beta \underline{y}+(1-\beta)(p-n+z)$. In the No-Gap case, the worker requests $w(p, n, z)=\beta(p+n-p-z)+(1-\beta)(n-z)=n-z$ and is left with zero payoffs, the firm accepts if $y \geq n-p-z$ and earns $p+y-w(p, n, z)=p+y-n+z$. So the outcome is different from that of one-sided repeated offers only in the Gap case.

The chance of trading $\xi(p-n)$ is the same as in the previous model, so it satisfies Condition 1 (c), and

$$
(r+\delta) \mathcal{G}(p-n)=\beta \int_{n-p-\underline{y}}^{\bar{z}}(p+\underline{y}+z-n) d F_{Z}(z) .
$$

Hence

$$
(r+\delta) \mathcal{G}^{\prime}(p-n)=\beta\left[1-F_{Z}(n-p-\underline{y})\right] \in(0, \xi(p-n)) .
$$

By the ex post efficiency of trade, this implies $(r+\delta) \mathcal{J}^{\prime}(p-n)=\xi(p-n)-(r+\delta) \mathcal{G}^{\prime}(p-n) \in$ $(0, \xi(p-n))$. Again, these are the desired properties and are symmetric. We conclude: 
Proposition 6. Under Assumption 1 the model of wage determination through quickly repeated alternating offers between an uninformed and an informed party, in stationary strategies and monotone beliefs, satisfies Conditions 1 and 2 and Increasing worker rents. Thus, both labor market volatility bounds of Propositions 1 and 2 apply.

\subsection{Two-Sided Private Information: Constrained Efficient Alloca- tion}

The theory of strategic bargaining in the presence of bilateral asymmetric information, whether with one-sided or alternating offers, has not produced compelling (unique) predictions based on the Perfect Bayes Nash equilibrium notion even after restricting the space of strategies by requirements such as stationarity of strategies and monotonicity of beliefs. The wealth of reasonable off-equilibrium beliefs simply sustains many robust equilibria, even after applying additional refinements.

We then turn to the constrained efficient allocation that maximizes expected gains from trade, as in Myerson and Satterthwaite (1983), henceforth MS83. This corresponds to the efficient allocation where both parties receive equal weight in the welfare function. Parties have access to a mediator, who receives announcements about the draws of private information, $y$ and $z$, and recommends a binding allocation: a trading decision and a wage. After learning their type, parties are willing to participate in the mechanism and truthfully reveal their types. In this wage negotiation context, the mediator enforcing the rules of the game can be thought of as an arbitrator of a labor dispute. The resulting constrained efficient allocation can be implemented with a multiplicity of payment functions, but importantly it produces a unique sharing of expected rents. This allocation is of great interest for its uniqueness, which allows us to apply our test, and for two additional reasons: it features the maximal expected gains from trade in the equilibrium of any unmediated bargaining game and, for some classes of belief distributions, it can be implemented through a sealed-bid double auction. In this subsection, we show that our weak bound from Proposition 1 applies to this allocation too. We have not been able to either establish or disprove Condition 2 and the strong bound.

The Mechanism Design Problem. A mediator, or principal, receives reports $\hat{y}$ and $\hat{z}$ by the two parties and enforces a probability of trade $x(\hat{y}, \hat{z}, p, n)$ and a wage $w(\hat{y}, \hat{z}, p, n)$ so as 
to maximize the sum of expected values to the two parties. The reports are a Bayesian Nash equilibrium of this optimal mechanism. That is, the efficient mechanism is a direct revelation game whose Bayesian Nash equilibrium produces the constrained efficient allocation.

Given a pair of reports $\hat{y}, \hat{z}$ and realizations $y, z$, the firm's value is

$$
(r+\delta) J(\hat{y}, \hat{z}, y, p, n)=p+y-w(\hat{y}, \hat{z}, p, n)
$$

and the worker's value is

$$
(r+\delta) W(\hat{y}, \hat{z}, z, p, n)=z+w(\hat{y}, \hat{z}, p, n)+\delta U=z+w(\hat{y}, \hat{z}, p, n)+\delta \frac{n}{r} .
$$

The constrained efficient allocation obtained through a direct revelation mechanism maximizes the total expected value to firm and worker

$$
\begin{aligned}
& \max _{x, w} \int_{\underline{z}}^{\bar{z}} \int_{\underline{y}}^{\bar{y}}[J(y, z, y, p, n)+W(y, z, z, p, n)] x(y, z, p, n) d F_{Y}(y) d F_{Z}(z) \\
& +\int_{\underline{z}}^{\bar{z}} \int_{\underline{y}}^{\bar{y}} U[1-x(y, z, p, n)] d F_{Y}(y) d F_{Z}(z)
\end{aligned}
$$

subject to (interim) Individual Rationality (IR) and Incentive Compatibility (IC) constraints of the firm: for all $y, \hat{y} \in[\underline{y}, \bar{y}]$

$$
\int_{\underline{z}}^{\bar{z}} J(y, z, y, p, n) x(y, z, p, n) d F_{Z}(z) \geq \max \left\langle 0, \int_{\underline{z}}^{\bar{z}} J(\hat{y}, z, y, p, n) x(\hat{y}, z) d F_{Z}(z)\right\rangle
$$

and of the worker: for all $z, \hat{z} \in[\underline{z}, \bar{z}]$

$$
\begin{aligned}
& \int_{\underline{y}}^{\bar{y}}\{W(y, z, z, p, n) x(y, z, p, n)+U[1-x(y, z, p, n)]\} d F_{Y}(y) \\
\geq & \max \left\langle U, \int_{\underline{y}}^{\bar{y}}\{W(y, \hat{z}, z, p, n) x(y, \hat{z})+U[1-x(y, \hat{z}, p, n)]\} d F_{Y}(y)\right\rangle .
\end{aligned}
$$

Notice that this is not a constrained efficient allocation for society: here parties take the outside option $n=r U$ as given, and just mind the division of rents. The objective function is independent of the wage $w$, which only plays the role of a transfer function to induce parties to truthfully reveal their valuations, thus only enters the IC and IR constraints. In the appendix we prove:

Lemma 7. Under Assumption 1, there exists a unique constrained efficient trading rule: trade iff $y \geq y^{*}(z, p-n)$ where the decreasing function $y^{*}=y^{*}(z, p-n)$ uniquely solves

$$
y^{*}+p-n+z=\frac{\mu}{1+\mu}\left\{\frac{1-F_{Y}\left(y^{*}\right)}{F_{Y}^{\prime}\left(y^{*}\right)}+\frac{1-F_{Z}(z)}{F_{Z}^{\prime}(z)}\right\}
$$


and $\mu \geq 0$ is the Lagrange multiplier on the constraint

$$
\int_{\underline{z}}^{\bar{z}} \int_{y^{*}(z, p-n)}^{\bar{y}}\left[p+y+z-n-\frac{1-F_{Y}(y)}{F_{Y}^{\prime}(y)}-\frac{1-F_{Z}(z)}{F_{Z}^{\prime}(z)}\right] d F_{Y}(y) d F_{Z}(z) \geq 0
$$

which is equivalent to all IC and IR constraints.

The ex post probability of trade is $x^{*}(y, z, p-n)=\mathbb{I}\left\{y \geq y^{*}(z, p-n)\right\}$ and the ex ante chance of trade, unconditional on private information, is

$$
\xi^{*}(p-n)=\int_{\underline{z}}^{\bar{z}}\left[1-F_{Y}\left(y^{*}(z, p-n)\right)\right] d F_{Z}(z)=\int_{\underline{y}}^{\bar{y}}\left[1-F_{Z}\left(z^{*}(y, p-n)\right)\right] d F_{Y}(y) .
$$

Corner solutions are encompassed: if $y^{*}(z)<\underline{y}$ for some worker type $z$, this and all higher types $z^{\prime}>z$ who like the job even better than $z$ are sure to trade. Conversely if $y^{*}(z)>\bar{y}$ for some worker type $z$, this and all lower types $z^{\prime}<z$ who like the job even less than $z$ are sure not to trade. Notice that the ex post efficient trading rule, trade iff $y+p-n+z \geq 0$, holds if and only if the constraint is not binding, or $\mu=0$. This happens in the Gap case, when $p-n$ is large enough that the supports of $p+y$ and $z-n$ are disjoint.

We can also state the constrained efficient trading rule in terms of the worker's private value: trade occurs iff $z \geq z^{*}(y, p-n)=y^{*-1}(y, p-n)$. Either way, the higher the valuation a party has for the match, the more likely she expects trade to be. By the implicit function theorem, these cutoff functions $y^{*}$ and $z^{*}$ are also differentiable in $p-n$. We now move to verify the conditions stated at the beginning of this Section 4.

Expected Rents. As shown in MS83, the expected value to each party, unconditional on trade but conditional on private information, can be written as

$$
\begin{aligned}
(r+\delta) \mathcal{G}^{*}(z \mid p, n) & =(r+\delta) \mathcal{G}^{*}(\underline{z} \mid p, n)+\int_{\underline{z}}^{z}\left[1-F_{Y}\left(y^{*}\left(z^{\prime}, p-n\right)\right)\right] d z^{\prime} \\
(r+\delta) \mathcal{J}^{*}(y \mid p, n) & =(r+\delta) \mathcal{J}^{*}(\underline{y} \mid p, n)+\int_{\underline{y}}^{y}\left[1-F_{Z}\left(z^{*}\left(y^{\prime}, p-n\right)\right)\right] d y^{\prime}
\end{aligned}
$$

Notice that $\mathcal{G}^{*}(z \mid p, n)$ is increasing in $z$ and $\mathcal{J}^{*}(y \mid p, n)$ is increasing in $y$, so the IR constraints $\mathcal{G}^{*}(z \mid p, n) \geq 0$ and $\mathcal{J}^{*}(y \mid p, n) \geq 0$ for each type of worker and firm are satisfied if they are for the lowest types $\underline{y}$ and $\underline{z}$. By Theorem 2 in MS83, these are binding at the optimum:

$$
\mathcal{G}^{*}(p, n \mid \underline{z})=\mathcal{J}^{*}(p, n \mid \underline{y})=0
$$


Taking expectations w.r. to private information, we obtain the expected values to each party unconditional on trade and on private information. In the second and fourth equalities we use integration by parts:

$$
\begin{aligned}
(r+\delta) \mathcal{G}^{*}(p-n) & =\int_{\underline{z}}^{\bar{z}} \int_{\underline{z}}^{z}\left[1-F_{Y}\left(y^{*}\left(z^{\prime}, p-n\right)\right)\right] d z^{\prime} F_{Z}^{\prime}(z) d z \\
& =\int_{\underline{z}}^{\bar{z}}\left[1-F_{Y}\left(y^{*}(z, p-n)\right)\right]\left[1-F_{Z}(z)\right] d z \\
& =\int_{\underline{z}}^{\bar{z}} \int_{\underline{y}}^{\bar{y}} F_{Y}^{\prime}(y) d y\left[1-F_{Z}(z)\right] x^{*}(y, z, p, n) d z \\
& =\int_{\underline{y}}^{\bar{y}} F_{Y}^{\prime}(y) \int_{z^{*}(y, p-n)}^{\bar{z}}\left[1-F_{Z}(z)\right] d z d y \\
& =\int_{\underline{y}}^{\bar{y}} \int_{z^{*}(y, p-n)}^{\bar{z}}\left(z-z^{*}(y, p-n)\right) F_{Z}^{\prime}(z) F_{Y}^{\prime}(y) d z d y
\end{aligned}
$$

Similarly

$$
(r+\delta) \mathcal{J}^{*}(p-n)=\int_{\underline{z}}^{\bar{z}} \int_{y^{*}(z, p-n)}^{\bar{y}}\left(y-y^{*}(z, p-n)\right) F_{Y}^{\prime}(y) F_{Z}^{\prime}(z) d z d y .
$$

Notice the analogy with the monopoly model: a trading party enjoys expected rents equal to the expected difference between its own valuation and the minimum acceptable valuation for trade.

These rents are uniquely defined by the trading rule $y^{*}$, that we proved to uniquely exist, and do not depend on the payment function $w^{*}$, which is defined residually. Therefore, $\mathcal{G}^{*}$ and $\mathcal{J}^{*}$ are uniquely defined as functions of $p-n$ only.

Verifying the Conditions. Lemma 7 immediately implies Condition 1 (c). In the Appendix, we prove the key result:

Lemma 8. $\mathcal{G}^{* \prime}(p-n) \geq 0$.

Since the roles of the worker and the firm in the mechanism design problem are symmetric, as long as both distributions of types satisfy Assumption 1, we also obtain $\mathcal{J}^{* \prime}(p-n) \geq 0$. Intuitively, when the average gains from trade $p-n$ rise, the mediator optimally re-adjusts his mechanism so as to spread the benefits across both parties. One might expect that, in situations where private information is lopsided and the IC constraint is much more binding for one party, larger gains from trade $p-n$ might be used by the mediator to relax that constraint 
and to greatly increase the chance of trade. This may require reducing the expected payoffs to the other party. Lemma 8 shows that this is impossible. Under bilateral asymmetric information satisfying Assumption 1, labor market fluctuations of the empirically observed magnitude are a symptom of wage-setting institutions that, albeit not necessarily privately inefficient in the Pareto sense, do not fully exploit all possible gains from trade.

Proposition 9. Under bilateral asymmetric information satisfying Assumption 1, the constrained efficient model of wage determination, in which a mediator maximizes ex ante expected gains from trade, satisfies Condition 1 and Increasing worker rents. Therefore, the labor market volatility bound of Proposition 1 applies.

\subsection{Summary}

There are essentially two kinds of allocations that emerge in the games that we considered. Either one party is left with no rents, as in the Coase conjecture, or an interior trading cutoff on private information is chosen to maximize the rents of one or both parties, as in the models of take-it-or-leave-it offers and the constrained efficient allocation. In the former case, our conditions apply trivially. In the latter, they follow in part from an implication of optimality, the envelope theorem.

The various models that we analyzed suggest a connection between private (in)efficiency of wage setting and labor market volatility. To this purpose, it is useful to describe the Pareto frontier under bilateral asymmetric information, interim participation and incentive compatibility constraints. Barring transfers between parties at the ex ante stage, the constrained efficient allocation is just one point on this frontier, right in the middle of it where both parties receive equal weights. The extreme points of the frontier correspond to take-it-orleave-it offers, where one party obtains all the weight. Although the entire frontier of this trading problem has not been fully characterized, in these three benchmark cases (points on the frontier) either one or both of our bounds apply. We conjecture that all the allocations on the frontier, for any given Pareto weights that do not change with aggregate productivity $p$, satisfy our conditions for volatility bounds. Verifying this conjecture is a task for future research. Some of the strategic bargaining models that we considered feature inefficiency but no amplification, so inefficiency in wage setting may be necessary but is certainly not sufficient 
for amplification of aggregate labor productivity shocks.

For belief distributions that give rise to linear "virtual valuations" (as defined in Assumption 1) the unique constrained efficient allocation can be implemented through a sealed-bid double auction (Satterthwaite and Williams 1989), the same mechanism as in Hall (2005), but cannot provide the desired amplification. Yet, the double auction with two-sided asymmetric information has multiple equilibria that are privately Pareto undominated, although they do not maximize average gains from trade. Therefore, Hall's mechanism, based on an appropriate selection from the set of Pareto undominated equilibria, might still deliver the desired wage rigidity and amplification with private information. Verifying this conjecture is difficult, for

two reasons. First, any amount of asymmetric information restricts the equilibrium set of the double-auction, relative to the latitude exploited by Hall in the complete information case. Second, the literature has not found a complete characterization of this equilibrium set. So we leave also this conjecture for future research.

\section{$5 \quad$ Transitory Aggregate Productivity Shocks}

We have justified our focus on permanent productivity shocks claiming that labor market volatility is even smaller if shocks are transitory. Thus the labor market volatility bounds should still apply if shocks are not fully permanent. In this section we show that this is the case, generalizing the labor market volatility bounds to an economy that switches between a high state $p^{H}$ and a low state $p^{L}$ of aggregate labor productivity. Transitions from the high state to the low state occur at rate $\lambda^{L}$, reverse transitions at rate $\lambda^{H}$. As an illustration we generalize the firm offer monopoly model to this environment and demonstrate that the bounds apply.

The equilibrium conditions now read

$$
\begin{aligned}
& \frac{c}{q\left(\theta^{H}\right)}=\mathcal{J}^{H}\left(p^{H}, n^{H}, p^{L}, n^{L}\right), \\
& \frac{c}{q\left(\theta^{H}\right)}=\mathcal{J}^{L}\left(p^{H}, n^{H}, p^{L}, n^{L}\right),
\end{aligned}
$$

for free entry and

$$
\begin{aligned}
& n^{H}=b+\theta^{H} q\left(\theta^{H}\right) \mathcal{G}^{H}\left(p^{H}, n^{H}, p^{L}, n^{L}\right) \\
& n^{L}=b+\theta^{L} q\left(\theta^{L}\right) \mathcal{G}^{L}\left(p^{H}, n^{H}, p^{L}, n^{L}\right) .
\end{aligned}
$$


for the opportunity cost of the worker. ${ }^{19} \mathrm{~A}$ model of wage determination is now a sextuple $\left\{G^{H}, G^{L}, J^{H}, J^{L}, x^{H}, x^{L}\right\}$ where for instance $G^{H}\left(y, z, p^{H}, p^{L}, n^{H}, n^{L}\right)$ is the initial worker rent from a match formed in a high aggregate state with idiosyncratic productivity component $y$ and amenity value $z$. Worker rents of matches created in the high state depend on $p^{L}$ and $n^{L}$ because labor productivity is no longer completely persistent and the match may eventually find itself in the low state. The probability of trade is now a more complicated object. Let $h(s)$ denote the history of a match of age $s$. It includes the history of the aggregate state and the history of trade within the match between match formation and the time at which it reaches age $s$. Then $x^{H}\left(y, z, p^{H}, n^{H}, p^{L}, n^{L}, h(s)\right)$ denotes the probability of trade at age $s$ after history $h(s)$. The sum $G^{H}+J^{H}$ is the joint present value of the match induced by the trade probability $x^{H}$. The ex ante functions $\mathcal{G}^{H}, \mathcal{G}^{L}, \mathcal{J}^{H}, \mathcal{J}^{L}$ are defined analogously to Equation (1). Thus $\mathcal{G}^{H}\left(p^{H}, n^{H}, p^{L}, n^{L}\right)$ is the ex ante worker rent of a match created in the high aggregate state. We make the natural assumption that for identical states $p^{H}=p^{L}$ and $n^{H}=n^{L}$ the model maps into the case we already studied: rents do not depend on the state in which the match is created and the probability of trade is independent of history, so we can define:

$$
\begin{aligned}
G(y, z, p, n) & \equiv G^{H}(y, z, p, n, p, n)=G^{L}(y, z, p, n, p, n) \\
J(y, z, p, n) & \equiv J^{H}(y, z, p, n, p, n)=J^{L}(y, z, p, n, p, n), \\
x(y, z, p, n) & \equiv x^{H}(y, z, p, n, p, n, h(s))=x^{L}(y, z, p, n, p, n, h(s)),
\end{aligned}
$$

and Equation (1) yields the corresponding ex ante functions $\mathcal{G}, \mathcal{J}$ and $\xi$.

For the case of permanent shocks we derived upper bounds on the elasticity of the $v$ - $u$ ratio with respect to aggregate labor productivity $p$. Analogously, here we derive upper bounds on the relative coefficient of variation of the $v$ - $u$ ratio and aggregate labor productivity $\frac{\sigma_{\theta}}{\sigma_{p}} \frac{\mu_{p}}{\mu_{\theta}}$. In the two state model the coefficient of variation for the $v$ - $u$ ratio can be written as

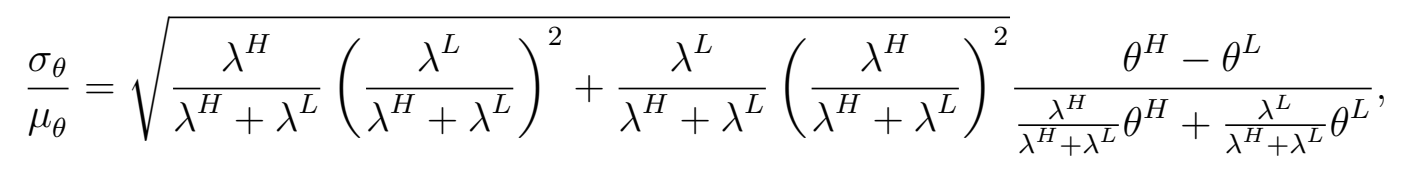

${ }^{19}$ In standard notation Equation (16) for the high state reads

$$
r U^{H}=b+\theta^{H} q\left(\theta^{H}\right) \mathcal{G}^{H}\left(p^{H}, n^{H}, p^{L}, n^{L}\right)+\lambda^{L}\left(U^{L}-U^{H}\right) .
$$

Thus here $n^{H}=r U^{H}-\lambda^{L}\left(U^{L}-U^{H}\right)$ and by symmetry $n^{L}=r U^{L}-\lambda^{H}\left(U^{H}-U^{L}\right)$. As before $\mathcal{G}^{H}\left(p^{H}, n^{H}, p^{L}, n^{L}\right)=\mathcal{W}^{H}\left(p^{H}, n^{H}, p^{L}, n^{L}\right)-U^{H}$ where $\mathcal{W}^{H}\left(p^{H}, n^{H}, p^{L}, n^{L}\right)$ is the utility of a worker in a job created in the high state. 
and an analogous formula holds for the coefficient of variation of productivity $\frac{\sigma_{p}}{\mu_{p}}$. Next we provide the natural generalization of Condition 1 for this environment.

Condition $\mathbf{1}^{\prime}$. The model of wage determination $\left\{G^{H}, G^{L}, J^{H}, J^{L}, x^{H}, x^{L}\right\}$ satisfies three properties:

(a) The partial effect of aggregate labor productivity in the high state $p^{H}$ on the difference in worker rents across states is non-negative

$$
\frac{\partial \mathcal{G}^{H}}{\partial p^{H}}-\frac{\partial \mathcal{G}^{L}}{\partial p^{H}} \geq 0
$$

(b) The partial effect of worker's opportunity cost in the high states $n^{H}$ on the difference in firm rents across states is non-positive

$$
\frac{\partial \mathcal{J}^{H}}{\partial n^{H}}-\frac{\partial \mathcal{J}^{L}}{\partial n^{H}} \leq 0
$$

(c) The trade probabilities $x^{H}$ and $x^{L}$ depend on $p^{H}$ and $n^{H}$ only through their difference $p^{H}-n^{H}$, on $p^{L}$ and $n^{L}$ only through their difference $p^{L}-n^{L}$, and are weakly increasing in both match-specific outcomes, the firm's idiosyncratic productivity $y$ and the worker's amenity value for the job $z$.

To see that this is a generalization of Condition 1, consider for instance part (a). Suppose productivity shocks are permanent. Then $\frac{\partial \mathcal{G}^{L}}{\partial p^{H}}=0$ : productivity in the high state is irrelevant for rents in the low state as the low state lasts forever. Moreover $\frac{\partial \mathcal{G}^{H}}{\partial p^{H}}=\frac{\partial \mathcal{G}}{\partial p^{H}}$ because the high state lasts forever. Thus Condition $1^{\prime}$ (a) reduces to Condition 1 (a) if productivity shocks are permanent.

\subsection{Weak Labor Market Volatility Bound}

To derive the weak bound we also need a generalization of Increasing worker rents.

Definition 1'. (Increasing Worker Rents) In a model of wage determination $\left\{G^{H}, G^{L}, J^{H}, J^{L}, x^{H}, x^{L}\right\}$ worker rents are Increasing if

$$
\frac{\partial \mathcal{G}^{H}}{\partial p^{H}}-\frac{\partial \mathcal{G}^{L}}{\partial p^{H}} \geq-\left(\frac{\partial \mathcal{G}^{H}}{\partial n^{H}}-\frac{\partial \mathcal{G}^{L}}{\partial n^{H}}\right) \geq 0 .
$$


Our approach to deriving the bounds in this setting is as follows. As before we consider small productivity shocks. Specifically, we linearize the model around a steady state with productivity $p$. That is, the two productivity values $p^{H}, p^{L}$ must satisfy $\frac{\lambda^{H}}{\lambda^{H}+\lambda^{L}} p^{H}+\frac{\lambda^{H}}{\lambda^{H}+\lambda^{L}} p^{H}=$ $p$. Importantly, with respect to the transition rates $\lambda^{H}$ and $\lambda^{L}$ we provide an upper bound, that is the bounds apply not only if $\lambda^{H}$ and $\lambda^{L}$ are close to zero, but for any values of $\lambda^{H}$ and $\lambda^{L}$.

First we note a fact that will be convenient in the linearization. In what follows all derivatives are evaluated at $(p, n, p, n)$ where $n$ is the steady state opportunity cost of the worker induced by productivity $p$. Identity (17) yields

$$
\frac{\partial \mathcal{J}^{H}}{\partial p^{H}}+\frac{\partial \mathcal{J}^{H}}{\partial p^{L}}=\frac{\partial \mathcal{J}^{L}}{\partial p^{H}}+\frac{\partial \mathcal{J}^{L}}{\partial p^{L}}
$$

and an analogous relationship holds among the derivatives of $\mathcal{G}^{H}$ and $\mathcal{G}^{L}$. These relationships allow us to compactly write the linearization of the model using only the derivatives with respect to productivity $p^{H}$ and opportunity $\operatorname{cost} n^{H}$ in the high state. Linearizing the model, we obtain

$$
(1-\eta) \mathcal{J} \frac{\theta^{H}-\theta^{L}}{\theta}=\left(\frac{\partial \mathcal{J}^{H}}{\partial p^{H}}-\frac{\partial \mathcal{J}^{L}}{\partial p^{H}}\right)\left(p^{H}-p^{L}\right)+\left(\frac{\partial \mathcal{J}^{H}}{\partial n^{H}}-\frac{\partial \mathcal{J}^{L}}{\partial n^{H}}\right)\left(n^{H}-n^{L}\right)
$$

from Equation (15) and

$$
n^{H}-n^{L}=\eta \frac{f}{\xi} \mathcal{G} \frac{\theta^{H}-\theta^{L}}{\theta}+\frac{f}{\xi}\left[\left(\frac{\partial \mathcal{G}^{H}}{\partial p^{H}}-\frac{\partial \mathcal{G}^{L}}{\partial p^{H}}\right)\left(p^{H}-p^{L}\right)+\left(\frac{\partial \mathcal{G}^{H}}{\partial n^{H}}-\frac{\partial \mathcal{G}^{L}}{\partial n^{H}}\right)\left(n^{H}-n^{L}\right)\right]
$$

from Equation (16). The second term on the right hand side of Equation (20) capture the change in worker rents. This leads to the following definition of procyclical worker rents for the linearized model:

Definition 2'. (Procyclical Worker Rents) Worker rents are Procyclical in equilibrium if

$$
\left(\frac{\partial \mathcal{G}^{H}}{\partial p^{H}}-\frac{\partial \mathcal{G}^{L}}{\partial p^{H}}\right)\left(p^{H}-p^{L}\right)+\left(\frac{\partial \mathcal{G}^{H}}{\partial n^{H}}-\frac{\partial \mathcal{G}^{L}}{\partial n^{H}}\right)\left(n^{H}-n^{L}\right) \geq 0
$$

In the appendix we prove the following version of Proposition 1 for the linearized model:

Proposition 1'. (Weak Volatility Bound) If the model of wage determination satisfies Condition $1^{\prime}$ and worker rents are Procyclical, then $\frac{\sigma_{\theta}}{\sigma_{p}} \frac{\mu_{p}}{\mu_{\theta}} \leq \bar{\varepsilon}_{\theta, W E A K}$. If worker rents are Increasing, then they are Procyclical. 


\subsection{Strong Labor Market Volatility Bound}

For the strong bound a generalization of Condition 2 is needed.

Condition $2^{\prime}$. In a model of wage determination $\left\{G^{H}, G^{L}, J^{H}, J^{L}, x^{H}, x^{L}\right\}$ firm rents satisfy the property

$$
(r+\delta)\left(\frac{\partial \mathcal{J}^{H}}{\partial p^{H}}-\frac{\partial \mathcal{J}^{L}}{\partial p^{H}}\right)(p, n, p, n) \leq \xi(p, n) .
$$

In the appendix we prove the following for the linearized model:

Proposition 2'. (Strong Volatility Bound) If the model of wage determination satisfies Conditions $1^{\prime}$ and $\mathscr{L}^{\prime}$ and if worker rents are Increasing, then $\frac{\sigma_{\theta}}{\sigma_{p}} \frac{\mu_{p}}{\mu_{\theta}} \leq \bar{\varepsilon}_{\theta, S T R O N G}$.

\subsection{Monopoly}

We consider take-it-or-leave-it offers by the firm and analyze the case without firm heterogeneity for simplicity. Again the firm has the power to commit not to make further offers to workers that have rejected an offer. It does not matter whether the firm also can commit not to make further offer to workers who accepted an offer. Thus we could assume that the firm makes a take-it-or-leave-it offer of a contract which specifies wage payments conditional on the history of the aggregate state, and has the ability to commit to this contract. Or we could let the firm change the wage whenever it finds it optimal to do so (but importantly, never makes a follow up offer to workers who reject a wage change). Both cases lead to the same outcome, which takes the following form. First consider a match created in the low state. Optimal firm offers yield the firm rent

$$
\begin{aligned}
&(r+\delta) \mathcal{J}^{L}\left(p^{H}, n^{H}, p^{L}, n^{L}\right)=\frac{1}{r+\delta+\lambda^{H}+\lambda^{L}} \\
& \cdot \max _{z}\left[\left(r+\delta+\lambda^{L}\right)\left(1-F_{Z}(z)\right)\left(p^{L}-n^{L}+z\right)+\lambda^{H}\left(1-F_{Z}(z)\right)\left(p^{H}-n^{H}+z\right)\right]
\end{aligned}
$$

To understand this expression, recall that in the absence of transitions between the two states, the firm would like to trade with more worker types in the high state. Here the firm starts in the low state, but expects the match to be in the high state for part of its duration. In this situation it is not optimal to hire workers only up to the point where profits in the low state $\left(1-F_{Z}(z)\right)\left(p^{L}-n^{L}+z\right)$ are maximized, because this constrains the set of worker types the firm can trade with once the boom comes. This consideration leads to a kind of labor hoarding: 
the cutoff chosen by the firm, denoted by $z_{M}^{L}\left(p^{H}-n^{H}, p^{L}-n^{L}\right)$, strikes a balance between the time the match spends in the two states, which is captured by the weights $r+\delta+\lambda^{L}$ and $\lambda^{H}$.

The situation is somewhat different for a match created in the high state. The reason is that hiring many worker types does not put a binding constraint on the set of worker types the firm wants to trade with once the slump comes, because in the slump the firm actually wants to shed worker types. Thus when a match is created in a boom, it is optimal to hire worker types up to the point where profits in the high state $\left(1-F_{Z}(z)\right)\left(p^{H}-n^{H}+z\right)$ are maximized. Let $z_{M}\left(p^{H}-n^{H}\right)$ denote the corresponding cutoff. Once the match enters a slump for the first time, it is essentially the same as a match created in the slump, and it is optimal to shed workers below the threshold $z_{M}^{L}\left(p^{H}-n^{H}, p^{L}-n^{L}\right)$. Thus the firm rent is given by

$$
\left(r+\delta+\lambda^{L}\right) \mathcal{J}^{H}\left(p^{H}, n^{H}, p^{L}, n^{L}\right)=\max _{z}\left[\left(1-F_{Z}(z)\right)\left(p^{H}-n^{H}+z\right)\right]+\lambda^{L} \mathcal{J}^{L}\left(p^{H}, n^{H}, p^{L}, n^{L}\right)
$$

This behavior of the firm generates worker rents

$$
\begin{aligned}
(r+\delta) \mathcal{G}^{L}\left(p^{H}, n^{H}, p^{L}, n^{L}\right) & =\int_{z_{M}^{b}\left(p^{H}-n^{H}, p^{L}-n^{L}\right)}^{\bar{z}}\left[z-z_{M}^{b}\left(p^{H}-n^{H}, p^{L}-n^{L}\right)\right] d F_{Z}(z) \\
\mathcal{G}^{H}\left(p^{H}, n^{H}, p^{L}, n^{L}\right) & =\frac{1}{r+\delta+\lambda^{L}} \int_{z_{M}\left(p^{H}-n^{H}\right)}^{\bar{z}}\left[z-z_{M}\left(p^{H}-n^{H}\right)\right] d F_{Z}(z) \\
& +\frac{\lambda^{L}}{r+\delta+\lambda^{L}} \mathcal{G}^{L}\left(p^{H}, n^{H}, p^{L}, n^{L}\right) .
\end{aligned}
$$

Having derived firm and worker rents for this model of wage determination, we now show that both the weak and the strong bound apply. Condition $1^{\prime}$ (c) is clearly satisfied, as trade follows a cutoff rule, and the cutoffs depend on $p^{H}, n^{H}, p^{L}$ and $n^{L}$ only trough the differences $p^{H}-n^{H}$ and $p^{L}-n^{L}$. Next we verify Condition $1^{\prime}$ (b) along with Condition $2^{\prime}$. We have

$$
(r+\delta) \frac{\partial \mathcal{J}^{L}}{\partial p^{H}}(p, n, p, n)=\frac{\lambda^{H}}{r+\delta+\lambda^{H}+\lambda^{L}}\left(1-F_{Z}\left(z_{M}(p-n)\right)\right)=\frac{\lambda^{H}}{r+\delta+\lambda^{H}+\lambda^{L}} \xi(p, n)
$$

where we exploit the fact that $z_{M}^{L}(p-n, p-n)=z_{M}(p-n)$. Differentiating $\mathcal{J}^{H}$ with respect to $p^{H}$ and combining results then yields

$$
(r+\delta)\left(\frac{\partial \mathcal{J}^{H}}{\partial p^{H}}-\frac{\partial \mathcal{J}^{L}}{\partial p^{H}}\right)(p, n, p, n)=\frac{r+\delta}{r+\delta+\lambda^{H}+\lambda^{L}} \xi(p, n) .
$$

This verifies Condition $2^{\prime}$. Recall that with permanent shocks Condition 2 holds with equality. Equation (21) is consistent with this result as the right hand side reduces to $\xi(p-n)$ for 
$\lambda^{H}=\lambda^{L}=0$. But with $\lambda^{H}>0$ or $\lambda^{L}>0$ there is some slack: fluctuations in firm rents across states and thereby labor market volatility are smaller if aggregate productivity shocks are more transient. Condition $1^{\prime}$ (b) immediately follows as $\frac{\partial \mathcal{J}^{H}}{\partial n^{H}}-\frac{\partial \mathcal{J}^{L}}{\partial n^{H}}=-\left(\frac{\partial \mathcal{J}^{H}}{\partial p^{H}}-\frac{\partial \mathcal{J}^{L}}{\partial p^{H}}\right)$.

It remains to show that worker rents are Increasing. Since $\frac{\partial \mathcal{G}^{H}}{\partial n^{H}}-\frac{\partial \mathcal{G}^{L}}{\partial n^{H}}=-\left(\frac{\partial \mathcal{G}^{H}}{\partial p^{H}}-\frac{\partial \mathcal{G}^{L}}{\partial p^{H}}\right)$ it is enough to show that $\frac{\partial \mathcal{G}^{H}}{\partial p^{H}}-\frac{\partial \mathcal{G}^{L}}{\partial p^{H}} \geq 0$. We have

$$
\left(\frac{\partial \mathcal{G}^{H}}{\partial p^{H}}-\frac{\partial \mathcal{G}^{L}}{\partial p^{H}}\right)(p, n, p, n)=\frac{\xi(p-n)}{r+\delta+\lambda^{L}}\left[\frac{\partial z_{M}^{L}}{\partial\left(p^{H}-n^{H}\right)}(p-n, p-n)-\frac{\partial z_{M}}{\partial(p-n)}(p-n)\right] .
$$

Rents in the high state respond more to an increase in $p^{H}$ than rents in the low state if the threshold $z_{M}$ declines more than the threshold $z_{M}^{L}$. Differentiating the first order condition implicitly defining $z_{M}^{L}$

$$
\frac{r+\delta+\lambda^{L}}{r+\delta+\lambda^{H}+\lambda^{L}}\left(p^{L}-n^{L}\right)+\frac{\lambda^{H}}{r+\delta+\lambda^{H}+\lambda^{L}}\left(p^{H}-n^{H}\right)+z_{M}^{L}=\frac{1-F_{Z}\left(z_{M}^{H}\right)}{f_{Z}\left(z_{M}^{H}\right)}
$$

with respect to $p^{H}$ yields

$$
\frac{\partial z_{M}^{L}}{\partial\left(p^{H}-n^{H}\right)}(p-n, p-n)=-\frac{\lambda^{H}}{r+\delta+\lambda^{H}+\lambda^{L}}\left[1-\left.\left(\frac{d}{d z} \frac{1-F_{Z}(z)}{f_{Z}(z)}\right)\right|_{z=z_{M}(p-n)}\right]^{-1} .
$$

Thus the response of $z_{M}^{L}$ is proportional to the fraction of time the match spends in the high aggregate state. Since $z_{M}$ does not put any weight on the low aggregate state it declines more strongly

$$
\frac{\partial z_{M}}{\partial(p-n)}(p-n)=-\left[1-\left.\left(\frac{d}{d z} \frac{1-F_{Z}(z)}{f_{Z}(z)}\right)\right|_{z=z_{M}(p-n)}\right]^{-1}
$$

and it follows that worker rents are Increasing.

Proposition $3^{\prime}$. Under Assumption 1 the model of wage determination through a take-it-orleave-it offer by the firm to a privately informed worker satisfies Conditions $1^{\prime}$ and $\mathscr{2}^{\prime}$ and Increasing worker rents. Thus, both labor market volatility bounds of Propositions $1^{\prime}$ and $\mathscr{L}^{\prime}$ apply.

\section{Conclusion}

In this paper we have made two contributions to the debate on whether modifications to the model of wage determination allow the search and matching model to generate larger labor market fluctuations in response to productivity shocks. First, we have shown that models of 
wage determination that cannot generate countercyclical rents, and thus at best generate rent rigidity, are subject to labor market volatility bounds which are quantitatively tight. The extent of amplification generated by rent rigidity is thus quite limited, and quantitatively rent rigidity is much closer to Nash bargaining than it is to wage rigidity. Second, for a sequence of classic models of wage determination under asymmetric information we have shown that rents are not countercyclical. Thus a model with asymmetric information in which merely rents are rigid cannot be the silver bullet which allows the search and matching model to match the magnitude of observed fluctuations in unemployment and vacancies. Allowing the model to do so solely through a modification of wage determination forces one to consider models that can generate countercyclical rents. On the other hand, rent rigidity can generate a limited but not insignificant amount of amplification. A full answer to Shimer's (2005) findings may involve more than one modification of the baseline model, of which a model of wage determination with rigid rents could be one.

\section{References}

Abowd, J.M., and F. Kramarz. 2003. "The Costs of Hiring and Separations." Labour Economics 10 (5): 499-530.

Barron, J. M., M. C. Berger, and D. A. Black. 1997. On-the-Job Training. Kalamazoo, MI: Upjohn Foundation for Employment Research.

Binmore, K., A. Rubinstein, and A. Wolinsky. 1986. "The Nash Bargaining Solution in Economic Modeling." RAND Journal of Economics 17 (2): 176-188.

Costain, J. S., and M. Reiter. 2005. "Business Cycles, Unemployment, and the Calibration of Matching Models." Working paper 872, Universitat Pompeu Fabra.

Grossman, S., and M. Perry. 1986. "Perfect Sequential Equilibrium." Journal of Economic Theory 39:97-119.

Guerrieri, V. 2006. "Heterogeneity and Unemployment Volatility." mimeo.

Gul, F., and H. Sonnenschein. 1988. "On Delay in Bargaining with One-Sided Uncertainty." Econometrica 56:601-11.

Gul, F. Sonnenschein, H., and R. Wilson. 1986. "Foundations of Dynamic Monopoly and the Coase Conjecture." Journal of Economic Theory 39:155-190. 
Hagedorn, M., and I. Manovskii. 2006. "The Cyclical Behaviour or Equilibrium Unemployment and Vacancies Revisited." mimeo.

Hall, R. E. 2005. "Employment Fluctuations with Equilibrium Wage Stickiness." American Economic Review 95 (1): 50-65.

Hall, R. E., and P. R. Milgrom. 2007. "The Limited Influence of Unemployment on the Wage Bargain." mimeo., Stanford University.

Hornstein, A., P. Krusell, and G. Violante. 2005. "Unemployment and Vacancy Fluctuations in the Matching Model: Inspecting the Mechanism." Economic Quarterly (Federal Reserve Bank of Richmond) 91:19-51.

Hosios, A. 1990. "On the Efficiency of Matching and Related Models of Search and Unemployment." Review of Economic Studies 57:279-298.

Kennan, J. 2006. "Private Information, Wage Bargaining and Employment Fluctuations." mimeo.

Menzio, G. 2005a. "A Cheap-Talk Theory of Random and Directed Search." mimeo.

—. 2005b. "High-Frequency Wage Rigidity." mimeo.

Mortensen, D. T., and E. Nagypal. 2005. "More on Unemployment and Vacancy Fluctuations." Working paper 11692, NBER.

Myerson, R. B., and M. A. Satterthwaite. 1983. "Efficient Mechanisms for Bilateral Trading." Journal of Economic Theory 29:265-281.

Petrongolo, B., and C. A. Pissarides. 2001. "Looking into the Black Box: A Survey of the Matching Function." Journal of Economic Literature 39:390-431.

Pissarides, C. A. 2000. Equilibrium unemployment theory. 2. Cambridge and London: MIT Press.

Satterthwaite, M. A., and S. R. Williams. 1989. "Bilateral Trade with the Sealed Bid k-Double Auction: Existence and Efficiency." Journal of Economic Theory 48:107-133.

Shapiro, C., and J. E. Stiglitz. 1984. "Equilibrium Unemployment as a Worker Discipline Device." American Economic Review 74 (3): 433-44.

Shimer, R. 2004. "The Consequences of Rigid Wages in Search Models." Journal of the European Economic Association 2 (2-3): 469-479.

—. 2005. "The Cyclical Behaviour of Equilibrium Unemployment and Vacancies." American Economic Review 95 (1): 25-49.

Silva, J., and M. Toledo. 2007. "Labor Turnover Cost and the Cyclical Behavior of Vacancies and Unemployment." mimeo. 


\section{A Appendix: Omitted Proofs}

Proof of Lemma 7. To solve this mechanism design problem, we appeal to MS83's formulation, and map our problem in their framework. Let $v \equiv p+y, \zeta \equiv n-z, \Phi(\zeta) \equiv 1-F_{Z}(n-\zeta)$, so $\Phi^{\prime}(\zeta) d \zeta=F_{Z}^{\prime}(n-\zeta) d \zeta=-F_{Z}^{\prime}(z) d z, \Gamma(v) \equiv F_{Y}(v-p)$, so $\Gamma^{\prime}(v) d v=F_{Y}^{\prime}(v-p) d v=$ $F_{Y}^{\prime}(y) d y$. Then the efficient mechanism maximizes expected gains from trade subject to IC, IR and budget balance.

$$
\begin{gathered}
\max _{x, w} \iint(v-\zeta) x(v, \zeta) d \Phi(\zeta) d \Gamma(y) \\
\text { s.t. } \int[v-w(v, \zeta)] x(v, \zeta) d \Phi(\zeta) \geq \max \left\langle 0, \int[v-w(\hat{v}, \zeta)] x(\hat{v}, \zeta) d \Phi(\zeta)\right\rangle \\
\int[w(v, \zeta)-\zeta] x(v, \zeta) d \Gamma(v) \geq \max \left\langle 0, \int[w(v, \hat{\zeta})-\zeta] x(v, \hat{\zeta}) d \Gamma(v)\right\rangle .
\end{gathered}
$$

This is the same formulation as in MS83. We apply their terminology and results. Let the "virtual types" be

$$
Q_{f}(v, \alpha) \equiv v-\alpha \frac{1-\Gamma(v)}{\Gamma^{\prime}(v)} \text { and } Q_{w}(\zeta, \alpha) \equiv \zeta+\alpha \frac{\Phi(\zeta)}{\Phi^{\prime}(\zeta)}
$$

which are, respectively, increasing in $v$ and decreasing in $\zeta$ by Assumption 1. Then IR, IC and budget balance are equivalent to

$$
\iint\left[Q_{f}(v, 1)-Q_{w}(\zeta, 1)\right] x(v, \zeta) d \Phi(\zeta) d \Gamma(y) \geq 0
$$

with equality if there is positive probability of no gains from trade (which we will assume to avoid trivialities).

Form a Lagrangian

$$
\max _{x} \iint\left\{v-\zeta+\mu\left[Q_{f}(v, 1)-Q_{w}(\zeta, 1)\right]\right\} x(v, \zeta) d \Phi(\zeta) d \Gamma(y)
$$

where $\mu$ is the multiplier. The FOC is

$$
x^{*}(v, \zeta)=\mathbb{I}\left\{v-\zeta+\mu\left[Q_{f}(v, 1)-Q_{w}(\zeta, 1)\right]>0\right\}=\mathbb{I}\left\{Q_{f}(v, M)>Q_{w}(\zeta, M)\right\}
$$

where $\mathbb{I}$ is the indicator function and

$$
M \equiv \frac{\mu}{1+\mu} \in[0,1]
$$

This, in particular, implies that trade occurs iff $v \geq \zeta$. More precisely, let the trading cutoff $v^{*}(\zeta, M)$ solve

$$
Q_{f}\left(v^{*}(\zeta, M), M\right)=Q_{w}(\zeta, M)
$$

so that trade occurs iff $v>v^{*}(\zeta, M)$, which is the same as $y \geq y^{*} \equiv v^{*}-p$.

Assumption 1 implies that $y^{*}$ is decreasing in $z$, and that $Q_{f}(., 1)$ and $Q_{w}(., 1)$ are increasing. Then $Q_{f}(v, M)$ and $Q_{w}(\zeta, M)$ are also increasing for every $M \in[0,1]$ (see MS83 who 
state this without proof; there is a simple proof by contradiction). It follows (MS83 Theorem 2 ) that an efficient mechanism exists, and the efficient rule is: trade iff $v>v^{*}(\zeta, M)$ for a cutoff function $v^{*}$ defined implicitly by

$$
v^{*}(\zeta, M)-\zeta=M\left\{\frac{1-\Gamma\left(v^{*}(\zeta, M)\right)}{\Gamma^{\prime}\left(v^{*}(\zeta, M)\right)}+\frac{\Phi(\zeta)}{\Phi^{\prime}(\zeta)}\right\} .
$$

Using our definitions, this is (13).

To show uniqueness, proceed by contradiction. Suppose that there exist two distinct efficient allocations $\left\{x_{i}^{*}\right\}_{i=1,2}$. Given the nature of the optimal rule (trade if $v>v^{*}(\zeta, M)$ ) these two mechanisms must be associated with two different values of the Lagrange multiplier, $M_{1}$ and $M_{2}>M_{1}$. Then $M_{2}>M_{1} \Leftrightarrow v^{*}\left(\zeta, M_{2}\right)>v^{*}\left(\zeta, M_{1}\right)$, which implies

$$
\begin{aligned}
& x_{2}^{*}(v, \zeta)=x_{1}^{*}(v, \zeta)=1 \text { for all } v>v^{*}\left(\zeta, M_{2}\right) \\
& x_{1}^{*}(v, \zeta)=1>0=x_{2}^{*}(v, \zeta) \text { for all } v \in\left(v^{*}\left(\zeta, M_{1}\right), v^{*}\left(\zeta, M_{2}\right)\right] \\
& x_{1}^{*}(v, \zeta)=x_{2}^{*}(v, \zeta)=0 \text { for all } v \leq v^{*}\left(\zeta, M_{1}\right)
\end{aligned}
$$

Therefore

$$
\iint(v-\zeta) x_{1}^{*}(v, \zeta) d \Phi(\zeta) d \Gamma(y)>\iint(v-\zeta) x_{2}^{*}(v, \zeta) d \Phi(\zeta) d \Gamma(y)
$$

so that the second mechanism, associated with the higher Lagrange multiplier, yields a strictly smaller objective function, and cannot be optimal.

Proof of Lemma 8. We use

$$
\frac{d y^{*}(z, p-n)}{d(p-n)}=-\frac{1-\frac{d \mu}{d(p-n)} \frac{1}{(1+\mu)^{2}}\left[\frac{1-F_{Y}\left(y^{*}(z, p-n)\right)}{F_{Y}^{\prime}\left(y^{*}(z, p-n)\right)}+\frac{1-F_{Z}(z)}{F_{Z}^{\prime}(z)}\right]}{1-\left.\frac{\mu}{1+\mu}\left(\frac{d}{d y} \frac{1-F_{Y}(y)}{F_{Y}^{\prime}(y)}\right)\right|_{y=y^{*}(z, p-n)}}
$$

and the analogous expression for $z^{*}$, which exist by the implicit function theorem.

There are two cases. First suppose that the Lagrange multiplier $\mu$ weakly declines in average gains from trade $p-n$. Notice that as $p-n \rightarrow \infty$ the incentive problem disappears, and $\mu$ eventually vanishes, so this case must occur for a sufficiently high value of $p-n$. Then the trading cutoff $z^{*}(y, p-n)$ or $y^{*}(z, p-n)$ strictly declines in $p-n$. From the above expression for the rents, it is immediate to show both parties strictly gain. Intuitively, when $\mu$ does not rise with $p-n$ the incentive constraint is no more severe, so trade becomes no less likely, and the planner still has additional gains from trade to distribute, so both parties gain.

Second, suppose that $\mu$ is (locally) strictly increasing in $p-n$. The IC constraint tightens, and trading may become less likely. By the envelope theorem, the maximized total expected gains from trade have derivative

$$
\mathcal{G}^{* \prime}(p-n)+\mathcal{J}^{* \prime}(p-n)=\frac{(1+\mu) \xi^{*}(p-n)}{(r+\delta)} .
$$


So the claim is equivalent to

$$
(1+\mu) \xi^{*}(p-n) \geq(r+\delta) \mathcal{J}^{* \prime}(p-n)=\int_{\underline{z}}^{\bar{z}}-\frac{d y^{*}(z, p-n)}{d(p-n)}\left[1-F_{Y}\left(y^{*}(z, p-n)\right)\right] F_{Z}^{\prime}(z) d z d y
$$

Using (14), it suffices to show that

$$
-\frac{d y^{*}(z, p-n)}{d(p-n)} \leq 1+\mu
$$

for all $z \in[\underline{z}, \bar{z}]$. By contradiction, suppose this inequality is reversed for some $\widehat{z} \in[\underline{z}, \bar{z}]$. Using (22) and multiplying through by the denominator of $d y^{*}(\widehat{z}, p-n) / d(p-n)$, which is positive by Assumption 1 (see MS83)

$$
\begin{aligned}
& -\frac{d \mu}{d(p-n)} \frac{1}{(1+\mu)^{2}}\left[\frac{1-F_{Y}\left(y^{*}(\widehat{z}, p-n)\right)}{F_{Y}^{\prime}\left(y^{*}(\widehat{z}, p-n)\right)}+\frac{1-F_{Z}(\widehat{z})}{F_{Z}^{\prime}(\widehat{z})}\right] \\
& >\mu\left[1-\left.\left(\frac{d}{d y} \frac{1-F_{Y}(y)}{F_{Y}^{\prime}(y)}\right)\right|_{y=y^{*}(\widehat{z}, p-n)}\right] .
\end{aligned}
$$

The RHS is positive by $\mu>0$ and by Assumption 1 . Therefore $d \mu / d(p-n)<0$, the desired contradiction.

Proof of Proposition 1'. The linearized counterpart of the formula for the coefficient of variation (18) is

$$
\frac{\sigma_{\theta}}{\mu_{\theta}}=\sqrt{\frac{\lambda^{H}}{\lambda^{H}+\lambda^{L}}\left(\frac{\lambda^{L}}{\lambda^{H}+\lambda^{L}}\right)^{2}+\frac{\lambda^{L}}{\lambda^{H}+\lambda^{L}}\left(\frac{\lambda^{H}}{\lambda^{H}+\lambda^{L}}\right)^{2}} \frac{\theta^{H}-\theta^{L}}{\theta} .
$$

Dividing by the corresponding formula for aggregate labor productivity yields

$$
\frac{\sigma_{\theta}}{\sigma_{p}} \frac{\mu_{p}}{\mu_{\theta}}=\frac{\theta^{H}-\theta^{L}}{p^{H}-p^{L}} \frac{p}{\theta}
$$

The proof proceeds in the same three steps followed in Section 3.1.

The first step is to show that $p^{H}-p^{L} \leq n^{H}-n^{L}$ implies $\theta^{H}-\theta^{L} \leq 0$. According to Condition $1^{\prime}$ (c), total rents in both aggregate states depend only on the differences $p^{H}-n^{H}$ and $p^{L}-n^{L}$. This implies

$$
\left(\frac{\partial \mathcal{J}^{H}}{\partial p^{H}}-\frac{\partial \mathcal{J}^{L}}{\partial p^{H}}\right)+\left(\frac{\partial \mathcal{J}^{H}}{\partial n^{H}}-\frac{\partial \mathcal{J}^{L}}{\partial n^{H}}\right)+\left(\frac{\partial \mathcal{G}^{H}}{\partial p^{H}}-\frac{\partial \mathcal{G}^{L}}{\partial p^{H}}\right)+\left(\frac{\partial \mathcal{G}^{H}}{\partial n^{H}}-\frac{\partial \mathcal{G}^{L}}{\partial n^{H}}\right)=0
$$

Using this relationship to eliminate $\left(\frac{\partial \mathcal{J}^{H}}{\partial n^{H}}-\frac{\partial \mathcal{J}^{L}}{\partial n^{H}}\right)$ from Equation (19) yields

$$
\begin{aligned}
(1-\eta) \mathcal{J} \frac{\theta^{H}-\theta^{L}}{\theta} & =\left[\left(\frac{\partial \mathcal{J}^{H}}{\partial p^{H}}-\frac{\partial \mathcal{J}^{L}}{\partial p^{H}}\right)+\left(\frac{\partial \mathcal{G}^{H}}{\partial p^{H}}-\frac{\partial \mathcal{G}^{L}}{\partial p^{H}}\right)\right]\left[\left(p^{H}-p^{L}\right)-\left(n^{H}-n^{L}\right)\right] \\
& -\left[\left(\frac{\partial \mathcal{G} g}{\partial p^{H}}-\frac{\partial \mathcal{G}^{L}}{\partial p^{H}}\right)\left(p^{H}-p^{L}\right)+\left(\frac{\partial \mathcal{G}^{H}}{\partial n^{H}}-\frac{\partial \mathcal{G}^{L}}{\partial n^{H}}\right)\left(n^{H}-n^{L}\right)\right] .
\end{aligned}
$$


If $\left(\frac{\partial \mathcal{J}^{H}}{\partial p^{H}}-\frac{\partial \mathcal{J}^{L}}{\partial p^{H}}\right) \leq 0$ it follows immediately from Equation (19) in conjunction with Condition $1^{\prime}$ (b) that $\theta^{H}-\theta^{L} \leq 0$. Otherwise the same implication follows from (19) together with Condition $1^{\prime}$ (a) and Procyclical worker rents.

The second step is to consider the case $p^{H}-p^{L}>n^{H}-n^{L}$. Using Equation (20), Procyclical worker rents imply

$$
n^{H}-n^{L} \geq \eta \frac{f}{\xi} \mathcal{G} \frac{\theta^{H}-\theta^{L}}{\theta} .
$$

Using the notation introduced in Section 3.1 this can be written as

$$
n^{H}-n^{L} \geq \eta f \frac{\theta^{H}-\theta^{L}}{\theta} \frac{1}{r+\delta+f}(w+\bar{z}-b) .
$$

Combining this with $n^{H}-n^{L} \leq p^{H}-p^{L}$ and using $\bar{z} \geq 0$ as well as $\bar{p} \geq p$ yields

$$
\frac{\theta^{H}-\theta^{L}}{p^{H}-p^{L}} \frac{p}{\theta} \leq \frac{\bar{p}}{\bar{p}-b} \frac{p-b}{w-b} \frac{1}{\eta} \frac{r+\delta+f}{f}=\bar{\varepsilon}_{\theta, W E A K} .
$$

The final step is to show that if worker rents are Increasing, then they are Procyclical. Suppose not. From the definition of Procyclicality it is clear that this can only occur if $p^{H}-p^{L}<$ $n^{H}-n^{L}$. Rewriting Equation (23) as

$$
\begin{aligned}
(1-\eta) \mathcal{J} \frac{\theta^{H}-\theta^{L}}{\theta} & =\left(\frac{\partial \mathcal{J}^{H}}{\partial p^{H}}-\frac{\partial \mathcal{J}^{L}}{\partial p^{H}}\right)\left[\left(p^{H}-p^{L}\right)-\left(n^{H}-n^{L}\right)\right] \\
& -\left[\left(\frac{\partial \mathcal{G}^{H}}{\partial p^{H}}-\frac{\partial \mathcal{G}^{L}}{\partial p^{H}}\right)+\left(\frac{\partial \mathcal{G}^{H}}{\partial n^{H}}-\frac{\partial \mathcal{G}^{L}}{\partial n^{H}}\right)\right]\left(n^{H}-n^{L}\right)
\end{aligned}
$$

reveals that $p^{H}-p^{L}<n^{H}-n^{L}$ together with Increasing worker rents implies that $\theta^{H} \leq \theta^{L}$. Consulting Equation (19), the supposition that worker rents are not Procyclical in conjunction with $\theta^{H} \leq \theta^{L}$ yields the contradiction that $n^{H} \leq n^{L}$.

Proof of Proposition $2^{\prime}$. Consider the case $n^{H}<n^{L}$. Given Increasing and thus Procyclical worker rents, it is clear from Equation (19) that this implies $\theta^{H}-\theta^{L} \leq 0$.

Next consider the case $n^{H} \geq n^{L}$. In this case Equation (25) implies

$$
\left(\frac{\partial \mathcal{J}^{H}}{\partial p^{H}}-\frac{\partial \mathcal{J}^{L}}{\partial p^{H}}\right)\left[\left(p^{H}-p^{L}\right)-\left(n^{H}-n^{L}\right)\right] \geq(1-\eta) \mathcal{J} \frac{\theta^{H}-\theta^{L}}{\theta} .
$$

Replacing $\mathcal{J}$ using the relationship $\mathcal{J}=\xi \frac{p-w}{r+\delta}$ and exploiting Condition $2^{\prime}$ yields

$$
\left(p^{H}-p^{L}\right)-\left(n^{H}-n^{L}\right) \geq(p-w)(1-\eta) \frac{\theta^{H}-\theta^{L}}{\theta} .
$$

Using (24) to eliminate $n^{H}-n^{L}$ and once again employing $\bar{z} \geq 0$ as well as $\bar{p} \geq p$ gives

$$
\frac{\theta^{H}-\theta^{L}}{p^{H}-p^{L}} \frac{p}{\theta} \leq \frac{\bar{p}}{\bar{p}-b}\left[\frac{\bar{p}-w}{\bar{p}-b} \frac{1}{\frac{1}{1-\eta}}+\frac{w-b}{\bar{p}-b} \frac{1}{\frac{1}{\eta} \frac{r+\delta+f}{f}}\right]^{-1}=\bar{\varepsilon}_{\theta, S T R O N G} .
$$

